\title{
Formation of temporally shaped electron bunches for beam-driven collinear wakefield accelerators
}

\author{
Wei Hou Tan $\odot,{ }^{1, *}$ Philippe Piot $\odot,{ }^{1,2, \dagger}$ and Alexander Zholents $\odot^{2, *}$ \\ ${ }^{1}$ Northern Illinois Center for Accelerator \& Detector Development and Department of Physics, \\ Northern Illinois University, DeKalb, Illinois 60115, USA \\ ${ }^{2}$ Argonne National Laboratory, Lemont, Illinois 60439, USA
}

(Received 19 January 2021; accepted 19 April 2021; published 20 May 2021)

\begin{abstract}
Beam-driven collinear wakefield accelerators (CWAs) that operate by using slow-wave structures or plasmas hold great promise toward reducing the size of contemporary accelerators. Sustainable acceleration of charged particles to high energies in the CWA relies on using field-generating relativistic electron bunches with a highly asymmetric current profile and a large energy chirp. A new approach to obtaining such bunches has been proposed and illustrated with the accelerator design supported by particle tracking simulations. It has been shown that the required particle distribution in the longitudinal phase space can be obtained without collimators, giving CWAs an opportunity for employment in applications requiring a high repetition rate of operation.
\end{abstract}

DOI: 10.1103/PhysRevAccelBeams.24.051303

\section{INTRODUCTION}

In a beam-based collinear wakefield accelerator (CWA), a high-charge drive bunch generates an electromagnetic field passing through a slow-wave structure (a dielectriclined or corrugated waveguide) or plasma. This field, called the wakefield, is used to accelerate a witness bunch propagating in the structure in the same direction behind the drive bunch [1-8]. An important figure of merit for a CWA is the transformer ratio, $\mathcal{R} \equiv\left|\mathcal{E}_{+} / \mathcal{E}_{-}\right|$, where $\mathcal{E}_{+}$is the maximum accelerating field behind the drive bunch, and $\mathcal{E}_{-}$is the maximum decelerating field within the drive bunch. For symmetric drive-bunch current distribution in time $I(t)$, the transformer ratio is limited to $\mathcal{R}<2$ [9]. However, asymmetric $I(t)$ can significantly enhance the transformer ratio [9], albeit at the expense of reduced $\mathcal{E}_{+}$ and $\mathcal{E}_{-}[10]$.

Bunch-shaping techniques for producing asymmetric current profile investigated hitherto include photocathode-laser intensity shaping [11-15], transverse-tolongitudinal phase-space exchange [16-20], control of longitudinal-phase-space nonlinearities [21-24], use of beam self-fields $[25,26]$, and interceptive method combined with local correlations [27-30]. Despite significant

\footnotetext{
*wtan1@niu.edu

ppiot@niu.edu

azholents@anl.gov
}

Published by the American Physical Society under the terms of the Creative Commons Attribution 4.0 International license. Further distribution of this work must maintain attribution to the author(s) and the published article's title, journal citation, and DOI. progress, the noninterceptive bunch-shaping techniques developed so far provide limited control over the current profiles and are not suitable for the production of bunches with an asymmetric current profile required for an efficient operation of wakefield accelerators. Consequently, producing drive bunches with an asymmetric current profile while preserving most of the bunch electrons has been an active research topic.

Another important consideration for a drive bunch arises from its proneness to the transverse beam-breakup (BBU) instability caused by the strong transverse forces due to the transverse wakefield [31-33]. A possible BBU-mitigation technique consists of imparting a large energy chirp along the drive bunch [34-36] and creating a current profile $I(t)$ that stimulates a dynamic adjustment of this chirp concurrently with the wakefield-induced bunch deceleration in the CWA [37].

The work reported in this paper was motivated by a design of a high repetition rate CWA for use in a freeelectron laser (FEL) facility described in Refs. [38,39]. This facility plans to employ up to ten FELs individually driven by a dedicated CWA. A single conventional accelerator delivers $\sim 1 \mathrm{GeV}$ drive electron bunches with a highly asymmetric $I(t)$ and a large energy chirp to the ten CWAs. Since the drive-bunch charge considered in $[38,39]$ is up to $10 \mathrm{nC}$ and the bunch repetition rate up to $500 \mathrm{kHz}$, the electron beam carries significant power. Therefore, using collimators to assist with the bunch shaping is prohibitive, and, consequently, preparing the drive bunches doing otherwise becomes a prime challenge.

To solve the problem, we undertook a new approach and distributed the task of obtaining the highly asymmetric $I(t)$ 
over the entire drive bunch accelerator beginning from the photocathode electron gun and ending by the final bunch compressor. To the best of our knowledge, our work demonstrates for the first time a pathway to producing electron bunches with complex current profiles without requiring the use of collimators. The employed technique is rather generic and can be adapted to other accelerator beam lines for preparing electron-bunch current distributions with profiles different than those considered in this paper. In the process, we implemented a backtracking technique [11] to search for possible beam line solutions. The advantage of the backtracking technique over usual (forward) tracking methods is that it simplifies the optimization process by first exploring beam line configurations that provide the final longitudinal phase-space (LPS) distribution while providing plausible input distribution. Such an approach is less constraining than a forward-tracking optimization where an initial distribution would have to be selected and parametrized. Once a set of beam line configurations and initial distributions have been found, a second step examines possible photoinjector arrangements that can provide phase-space distributions close to the one obtained via backtracking.

Although the main focus of the work was to obtain a drive bunch with the required distribution in the longitudinal phase space (LPS), an equally important additional objective was to ensure the associated transverse emittances commensurate with the small-aperture CWA.

\section{THE DRIVE BUNCH AND THE WAKEFIELD}

We define the longitudinal charge distribution in the electron bunch as $q(z)$ and consider bunches localized on the interval $0 \leq z \leq L$, where $z$ is the distance behind the bunch head. Therefore, we have

$$
\int_{0}^{L} q(z) \mathrm{d} z=Q
$$

where $Q$ is the total bunch charge. Following [10], we use the Green's function $G(z)$ consisting only of a fundamental mode $^{1}$

$$
G(z)=2 \kappa_{\|} \cos (k z) H(z)
$$

where $\kappa_{\|}$is the loss factor of a point particle per unit length, $k=2 \pi / \lambda$ is the wave vector, $\lambda$ is the wavelength, and $H(z)$ is the Heaviside step function. The longitudinal electric field within the electron bunch can be written as $[40,41]$

$$
\mathcal{E}_{-}(z)=2 \kappa_{\|} \int_{0}^{z} \cos \left[k\left(z-z^{\prime}\right)\right] q\left(z^{\prime}\right) \mathrm{d} z^{\prime}, \quad z \leq L,
$$

which is a Volterra equation of the first kind for the function $q(z)$ with the trigonometric kernel $\cos \left[k\left(z^{\prime}-z\right)\right]$. If we assume that $\mathcal{E}_{-}(0)=0$ at the bunch head, then the solution of Eq. (3) is given by [42]

$$
q(z)=\frac{1}{2 \kappa_{\|}}\left[\mathcal{E}_{-}^{\prime}(z)+k^{2} \int_{0}^{z} \mathcal{E}_{-}(x) \mathrm{d} x\right]
$$

where $\mathcal{E}_{-}(z)$ is a known function, and its derivative is taken over $z$. Hence, $q(z)$ is defined.

In order to maintain the stability of the drive bunch in the CWA throughout its deceleration, we require the bunch's relative chirp to be constant while being decelerated by the wakefield $\mathcal{E}_{-}(z)$, based on studies in [37]. This requirement is achieved by having a small linear variation in energy loss within the bunch, where head particles lose more energies than tail particles such that

$$
\chi(s)=\frac{1}{E_{0}(\mathrm{~s})} \frac{\partial E}{\partial z} \propto \mathcal{E}_{-}^{\prime}(z) \equiv \mathrm{const}
$$

where $E_{0}(\mathrm{~s})$ is the energy of the reference particle, and $s$ is the distance propagated by the bunch in the CWA. This is accomplished by using the electron bunch producing $\mathcal{E}_{-}$ with a linear variation in $z$. Similar to Ref. [9], we solve Eq. (4) considering $q(z)$ to be constant in the range $0 \leq$ $z<\xi$ with $\xi=\frac{1}{k} \arccos (\chi / k)$, in which case the continuities of $\mathcal{E}_{-}(z)$ and $\mathcal{E}_{-}^{\prime}(z)$ are preserved over the entire bunch length

$$
\begin{aligned}
q(z) & = \begin{cases}q_{0}, & 0 \leq z<\xi, \\
q_{0}\left\{1-k \xi \sin (k \xi)+\frac{k^{2}}{2} \xi^{2} \cos (k \xi)+\left[k \sin (k \xi)-k^{2} \xi \cos (k \xi)\right] z+\frac{k^{2}}{2} \cos (k \xi) z^{2}\right\}, & \xi \leq z \leq L,\end{cases} \\
\text { with } \quad q_{0} & =\frac{6 Q}{6 L+k^{2} \cos (k \xi)(L-\xi)^{3}+3 k \sin (k \xi)(L-\xi)^{2}} .
\end{aligned}
$$

Setting $\chi=0$ simplifies $q(z)$ to one used in [9]. Figure 1 shows examples of doorstep distributions with an

\footnotetext{
${ }^{1}$ It has been shown in [10] that a multimode Green's function is less effective in producing a high transformer ratio.
}

associated wakefield calculated for $L=\lambda$, with $\chi=0$ and $\chi=-\frac{1}{10 \lambda}$. In this example we considered a corrugated waveguide with radius $a=1 \mathrm{~mm}$ and fundamental mode frequency $f=180 \mathrm{GHz}$, as discussed in Ref. [43]. The current profile has sharp features that are challenging to 


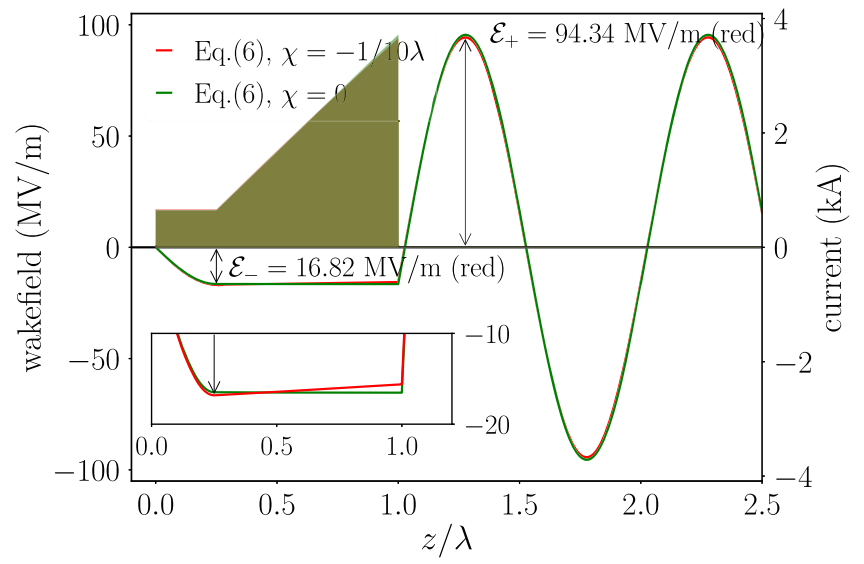

FIG. 1. Doorstep distributions described by Eq. (6) (shaded areas) with associated wakefields (traces) calculated for a bunch length $L=\lambda$, with $\chi=0$ (green trace) and $\chi=-\frac{1}{10 \lambda}$ (red trace), where transformer ratios are 5.8 and 5.6 respectively. The wakefields are computed for a bunch charge of $10 \mathrm{nC}$ and use a single-mode Green's function [Eq. (2)] with $f=180 \mathrm{GHz}$ and $\kappa_{\|}=14.3 \mathrm{kV} / \mathrm{pC} / \mathrm{m}$ calculated using ECHO [44].

realize. Consequently, the distribution defined by Eq. (6) is used only as a starting point to construct a practically realizable distribution shown in Fig. 2 with similar final properties listed in Table I. Specifically, the distribution is obtained by applying a Hann-window filter on the distribution described by Eq. (6) to smooth its sharp edges.

\section{A PRELIMINARY DESIGN OF THE DRIVE BUNCH ACCELERATOR}

\section{A. Basic considerations}

A block diagram of the drive bunch accelerator is shown in Fig. 3. It utilizes a commonly used configuration (see, for example, $[45,46])$ and includes a photocathode-gun-based injector, three linac sections, and two bunch compressors. Linac sections L1 and L2 are based on $650 \mathrm{MHz}$ superconducting (SRF) linac structures, and linac section L39 is based on $3.9 \mathrm{GHz}$ SRF structures. It is used for linearization of the electron distribution in the longitudinal phase space (LPS). The beamline also incorporates two bunch

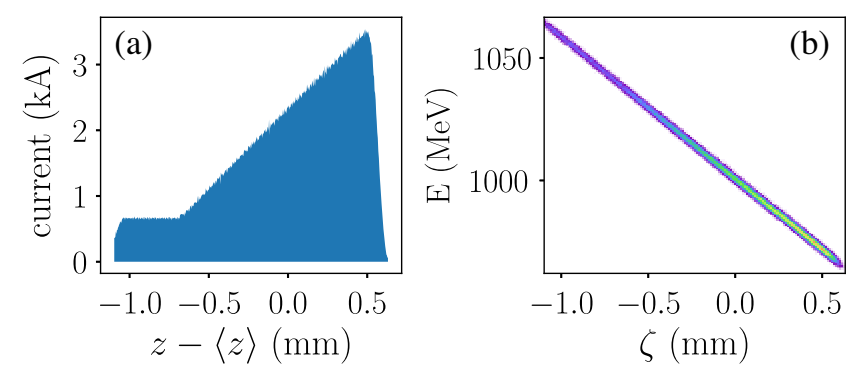

FIG. 2. A target drive bunch peak current (a) and longitudinal phase space (b) distributions at the end of the drive bunch accelerator.
TABLE I. Main parameters associated with the drive bunch distribution shown in Fig. 2.

\begin{tabular}{lcc}
\hline \hline Bunch parameter & Value & Unit \\
\hline Charge & 10 & $\mathrm{nC}$ \\
Reference energy & 1 & $\mathrm{GeV}$ \\
rms length & 422 & $\mu \mathrm{m}$ \\
Peak current & 3.5 & $\mathrm{kA}$ \\
rms fractional energy spread & 2.44 & $\%$ \\
rms fractional slice energy spread & 0.1 & $\%$ \\
\hline \hline
\end{tabular}

compressors labeled as $\mathrm{BC} 1$ and $\mathrm{BC} 2$ in Fig. 3. Here we take advantage of the requirement to prepare the drive bunch with the energy chirp seen in Fig. 2(b) and move $\mathrm{BC} 2$ to the end of the linac, since we do not need to use the linac to remove the energy chirp after bunch compression.

Using the known, target LPS distribution $\Phi_{f}\left(z_{f}, E_{f}\right)$ at the end of the accelerator shown in Fig. 2, we performed the one-dimensional (1D) backward tracking proposed in [11] to find the LPS distribution $\Phi_{i}\left(z_{i}, E_{i}\right)$ at the entrance of L1. We stopped at L1 where the beam energy is approximately $50 \mathrm{MeV}$ considering that $1 \mathrm{D}$ tracking may not be reliable at lower energies where transverse and longitudinal space charge effects are stronger. The assumption is that at this point the backward tracking will produce a plausible $\Phi_{i}\left(z_{i}, E_{i}\right)$ that can be matched by the injector. Specifically, we constrained the peak current to $I \approx 300 \mathrm{~A}$ and sought $\Phi_{i}\left(z_{i}, E_{i}\right)$ with minimal high-order correlations.

A tracking program, TWICE [47], was developed for rapid prototyping of the longitudinal dynamics in the linac without accounting for a transverse motion. The program adopts an approach similar to that used in LiTrack [48]. An important feature of TWICE is its ability to perform backward tracking including time reversal of the collective effect, see the Appendix.

The physics model implemented in TWICE includes the geometric wakefields in the accelerating sections, longitudinal space charge effects (LSCs), and coherent synchrotron radiation (CSR). The Green's functions needed for modeling of the geometric wakefield effects in the $650 \mathrm{MHz}$ and $3.9 \mathrm{GHz}$ linac sections were computed using the ECHO software and the empirical formula documented in Ref. [49].

The backward tracking was performed to define $\Phi_{i}\left(z_{i}, E_{i}\right)$ using $\Phi_{f}\left(z_{f}, E_{f}\right)$, shown in Fig. 2. The following constraints for the accelerator components were observed. First, the BBU-mitigation scheme implemented in the CWA requires a drive bunch with the negative chirp $\frac{\partial E}{\partial z}<0$, which implies that the longitudinal dispersions of BC1 and BC2 should be $R_{56}^{(1)}>0$ and $R_{56}^{(2)}>0$, as we

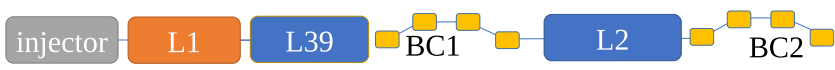

FIG. 3. Block diagram of the drive bunch accelerator. 
want to maintain a negative chirp throughout the entire accelerator. Second, a total energy gain of $\sim 950 \mathrm{MeV}$ in the linac part after the injector is needed. Third, an overall compression factor of $\sim 10$ is required from two bunch compressors.

In order to enforce all these constraints, TWICE was combined with the multiobjective optimization framework DEAP [50]. The optimization was performed by analyzing the LPS distributions upstream of $\mathrm{BC} 1$ and $\mathrm{L} 1$ to extract the central energy of the beam slices at every $z$-coordinate and to fit the slice-energy dependence on $z$ with the polynomial

$$
E(z)=c_{0}+c_{1} z+c_{2} z^{2}+c_{3} z^{3},
$$

where $c_{i}$ are constants derived from the fit. The optimizer objective function is to minimize the ratio $c_{2} / c_{1}$ at both locations. The third order coefficient $c_{3}$ was not optimized as we limited the 1D-tracking simulation to second order; see Appendix.

\section{B. Discussion of 1D simulation results}

A list of optimized accelerator settings found with TWICE backward tracking is given in Table II and the resulting $\Phi_{i}\left(z_{i}, E_{i}\right)$ is shown in Figs. 4(a) and 4(b). The forward tracking using this distribution recovers $\Phi_{f}\left(z_{f}, E_{f}\right)$, as seen in Figs. 4(c) and 4(d). The excellent agreement between Figs. 2(a) and 2(b) and Figs. 4(c) and 4(d) demonstrates the ability of TWICE to properly handle collective effects in both forward and backward tracking.

Each accelerator component serves a special role in obtaining the above-shown result. Linac section L1 provides energy gain and operates far from the crest acceleration to produce the required negative chirp. Linac section L39 corrects a second-order correlation between $E$ and $z$ imprinted on the bunch by the injector and L1 before it enters BC1. Linac section L2 operates even further off-crest to impart the necessary large chirp required for

TABLE II. Optimized parameters from the one-dimensional model.

\begin{tabular}{lcc}
\hline \hline Parameter & Value & Unit \\
\hline Accelerating voltage L1 & 219.46 & $\mathrm{MV}$ \\
Phase L1 & 17.81 & $\mathrm{deg}$ \\
Frequency L1 & 650 & $\mathrm{MHz}$ \\
Accelerating voltage L39 & 9.57 & $\mathrm{MV}$ \\
Phase L39 & 205.72 & $\mathrm{deg}$ \\
Frequency L39 & 3.9 & $\mathrm{GHz}$ \\
$R_{56}$ for bunch compressor 1 (BC1) & 0.1321 & $\mathrm{~m}$ \\
$T_{566}$ for bunch compressor 1 (BC1) & -0.1581 & $\mathrm{~m}$ \\
Accelerating voltage L2 & 847.69 & $\mathrm{MV}$ \\
Phase L2 & 28 & $\mathrm{deg}$ \\
Frequency L2 & 650 & $\mathrm{GHz}$ \\
$R_{56}$ for bunch compressor 2 (BC2) & 0.1301 & $\mathrm{~m}$ \\
$T_{566}$ for bunch compressor 2 (BC2) & 0.22 & $\mathrm{~m}$ \\
\hline \hline
\end{tabular}
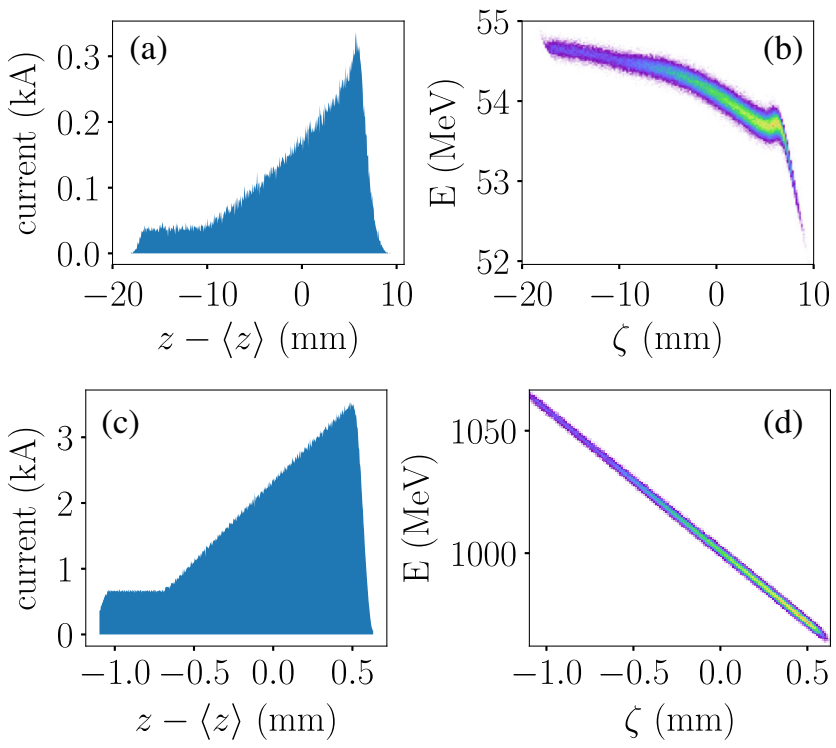

FIG. 4. Current (a) and (c) and LPS (b) and (d) distributions obtained from the backward-tracking optimization (a) and (b) and forward-tracked up BC2 end (c,) and (d) to confirm the agreement with the targeted distribution shown in Fig. 2.

maintaining beam stability in the CWA. Both bunch compressors shorten the bunch lengths and impact the LPS distributions. The values of $T_{566}$ selected in both bunch compressors ensure achieving $\Phi_{f}\left(z_{f}, E_{f}\right)$ despite the large energy chirp. The use of a negative $T_{566}$ in $\mathrm{BC} 1$ and a positive $T_{566}$ in $\mathrm{BC} 2$ enables the generation of a doorsteplike initial distribution without giving rise to a current spike, where $T_{566}$ has the effect of shifting the peak of current $[21,51]$. In this paper, we adopt the convention that $T_{566}$ with a negative (respectively positive) sign shifts the peak of current distribution to the tail (respectively head).

The result of the backward-tracking optimization provides only a starting point for obtaining a more realistic solution. For instance, the zigzag feature observed in the tail of the LPS distribution in Fig. 4(b) is challenging to create. In the following sections, we discuss how 1D backward-tracking results guide the design of a photocathode-gun-based injector and the downstream accelerator lattice.

\section{INJECTOR DESIGN}

Given the required initial LPS distribution obtained from the backward tracking, the next step is to explore whether such LPS distribution is achievable downstream of the injector; our approach relies on temporally shaping the photocathode laser pulse [52].

The injector beam line was modeled using the particlein-cell beam-dynamics program ASTRA, which includes a quasistatic space-charge algorithm [53]. The program was combined with the DEAP multivariate optimization framework to find a possible injector configuration and the laser 
pulse shape that realize the desired final bunch distribution while minimizing the transverse-emittance downstream of the photoinjector.

The injector configuration consists of a $200 \mathrm{MHz}$ quarter-wave SRF gun [54-56], coupled to a $650 \mathrm{MHz}$ accelerator module composed of five 5-cell SRF cavities [57]. The gun includes a high- $\mathrm{T}_{c}$ superconducting solenoid [58] for emittance control.

In the absence of collective effects, the photoemitted electron-bunch distribution mirrors the laser pulse distribution. In practice, image-charge and space-charge effects are substantial during the emission process and distort the electron bunch distribution. Consequently, devising laserpulse distributions that compensate for the introduced deformities is critical to the generation of bunches with tailored current profiles. The laser pulse distribution is characterized by $I(t, r)=\Lambda(t) R(r)$, where $\Lambda(t)$ and $R(r)$ describe the laser temporal profile and the transverse envelope, respectively. In our simulation, we assumed the transverse distribution to be radially uniform $R(r)=H\left(r_{c}-r\right)$, where $H\left(r_{c}-r\right)$ is Heaviside step function and $r_{c}$ is the maximum radius. The temporal profile is parametrized as

$$
\begin{aligned}
& \Lambda(t)=A f(t) S[a(t-f)] S[-b(t-g)], \\
& f(t)= \begin{cases}h, & \text { where } \\
h+d(t-c)^{d-1}, & c \leq t \leq 1 \\
0, & \text { elsewhere }\end{cases}
\end{aligned}
$$

where $A$ is the normalization constant; and $a, b c, d, f, g$, and $h$ are the parameters controlling the bunch shape. The smooth edges at both ends are characterized by $a, b, f, g$ via the logistic function $S(u)=1 /\left(1+\mathrm{e}^{-u}\right) ; c$ determines the length of the constant part of the laser pulse analogous to the length of the bunch head of the doorstep distribution; and $h$ determines the relative amplitude of the constant laser pulse; see Fig. 5. The overall shape resembles a smoothed version of the doorstep distribution. The lasershape parameters introduced in Eq. (8), the laser spot size, the phase and accelerating voltage of all $\mathrm{rf}$ cavities, and the high-temperature superconductor solenoid peak magnetic field were taken as control parameters for the optimization algorithm. The beam kinetic energy was constrained not to exceed $60 \mathrm{MeV}$. In order to quantify the final distribution, we used the Wasserstein's distance [59] to quantify how close the shape of the simulated macroparticle distribution at the injector exit $I^{(o)}(z)$ was to the shape of the target macroparticle density distributions $I^{(t)}(z)$ obtained from backward-tracking results. Specifically, the Wasserstein's distance is evaluated as

$$
\mathcal{D}=\sum_{i=1} N_{b} \frac{\left\|I_{i}^{(t)}-I_{i}^{(o)}\right\|}{N_{b}},
$$

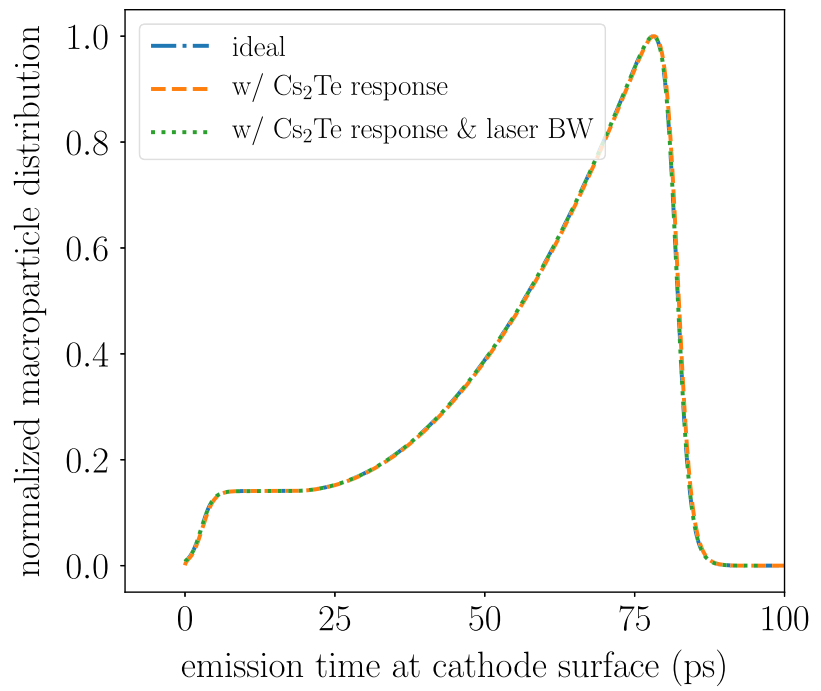

FIG. 5. Programmed macroparticle distributions at the photocathode surface: for an optimized laser pulse (blue trace), taking into account the photocathode response (orange trace), and both the cathode response and finite bandwidth (BW) of the laser pulse (green trace). The laser bandwidth is taken to be $\delta f=2 \mathrm{THz}$.

where $I_{i}^{(t, o)}$ are the corresponding histograms of the macroparticles' longitudinal positions over the interval $i$ defined as $\left[z_{i}+\delta z, z_{i}-\delta z\right]$, with $\delta z \equiv \frac{\max (z)-\min (z)}{N_{b}}$ being the longitudinal bin size and $N_{b}$ the number of bins used to compute the histogram. Additionally, we need to have a small beam transverse emittance. Hence, the Wasserstein's distance and the beam transverse emittance were used as our objective functions to be minimized.

An example of the optimized injector settings is summarized in Table III, and the evolution of the associated beam parameters along the beam line is presented in Figs. 6 and 7. The final bunch distributions $11.5 \mathrm{~m}$ downstream of the photocathode appears in Fig. 6. The beam transverse phase space indicates some halo population. Ultimately, an alternative laser-shaping approach implementing a spatiotemporal-tailoring scheme could provide better control over the transverse emittance while producing the required shaped electron beams [52]. We also find, as depicted in Fig. 6, that the current distribution tends to have a peak current lower than that desired from the backward-tracking result shown in Fig. 4. Although higher currents are possible, they come at the expense of transverse emittance. Consequently, the distribution generated from the injector was considered as an input to the one-dimensional forward tracking simulations. Iterations of one-dimensional forward tracking simulation studies were done to further crosscheck accelerator parameters needed for the beam-shaping process. We especially found that the desired final bunch shape at $1 \mathrm{GeV}$ can be recovered by altering the L39 phase and amplitude.

Furthermore, the small rms slice energy spread $\sigma_{E}<$ $10 \mathrm{keV}$ simulated from the injector [see Fig. 4(b)] is 
TABLE III. Optimized parameters for the injector and beam parameters at $s=11.67 \mathrm{~m}$ from the photocathode surface. The rf-cavity phases are referenced with respect to the maximumenergy phases.

\begin{tabular}{lcc}
\hline \hline Parameter & Value & Unit \\
\hline Laser spot radius & 2.810 & $\mathrm{~mm}$ \\
Laser duration & 91 & $\mathrm{ps}$ \\
rf gun peak electric field & 40 & $\mathrm{MV} / \mathrm{m}$ \\
rf gun phase & 1.71 & $\mathrm{deg}$ \\
Cavity C1 peak electric field & 13.25 & $\mathrm{MV} / \mathrm{m}$ \\
Cavity C1 phase & 11.28 & $\mathrm{deg}$ \\
Cavity C2 phase & -15.05 & $\mathrm{deg}$ \\
Cavities C2 to C5 peak electric field & 20 & $\mathrm{MV} / \mathrm{m}$ \\
Cavities C3 to C4 phase & 0 & $\mathrm{deg}$ \\
Cavity C5 phase & 20 & $\mathrm{deg}$ \\
Cavity C1 distance from the photocathode & 2.67 & $\mathrm{~m}$ \\
Solenoid B-field & 0.2068 & $\mathrm{~T}$ \\
Shape parameter $a$ & 93.55 & $\ldots$ \\
Shape parameter $b$ & 80.70 & $\ldots$ \\
Shape parameter $c$ & 0.196 & $\ldots$ \\
Shape parameter $d$ & 3.044 & $\ldots$ \\
Shape parameter $f$ & 0.030 & $\ldots$ \\
Shape parameter $g$ & 0.900 & $\ldots$ \\
Shape parameter $h$ & 0.207 & $\ldots$ \\
Final beam energy & 58.7 & $\mathrm{MeV}$ \\
Final beam bunch length & 7.06 & $\mathrm{~mm}$ \\
Final beam transverse emittance & 8.36 & $\mu \mathrm{m}$ \\
Final beam rms radius & 1.64 & $\mathrm{~mm}$ \\
\hline \hline
\end{tabular}

responsible for the formation of local current spikes which result in significant CSR-induced phase-space distortions in addition to possibly rendering the bunch susceptible to
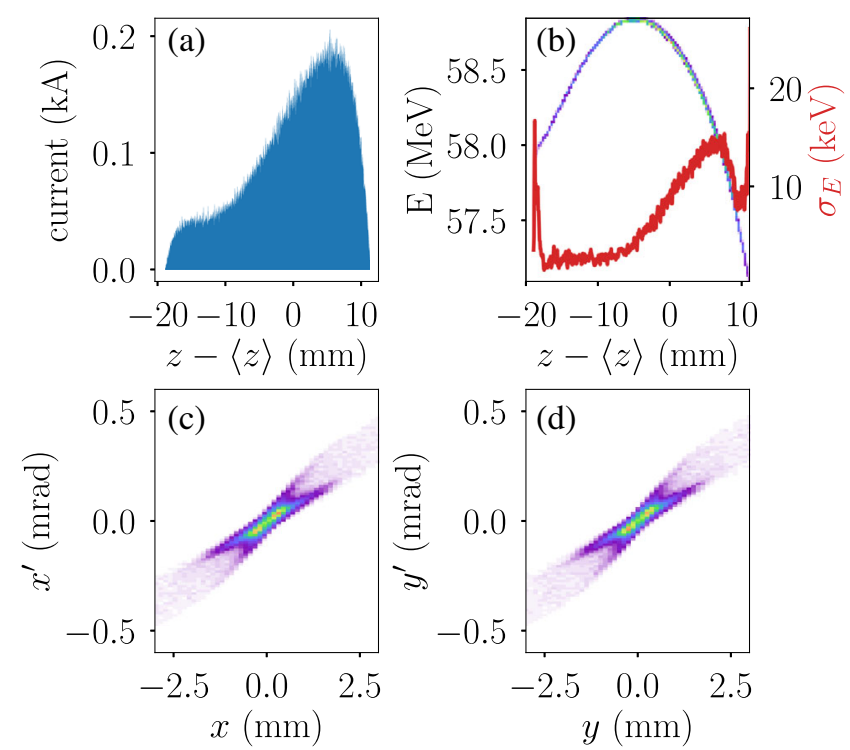

FIG. 6. Current profile (a) with associated LPS (b), and horizontal (c) and vertical (d) phase-space distributions simulated with ASTRA at the end of the photoinjector $(11.67 \mathrm{~m}$ from the photocathode). In plot (b), the red trace represents the slice rms energy spread $\sigma_{E}$.
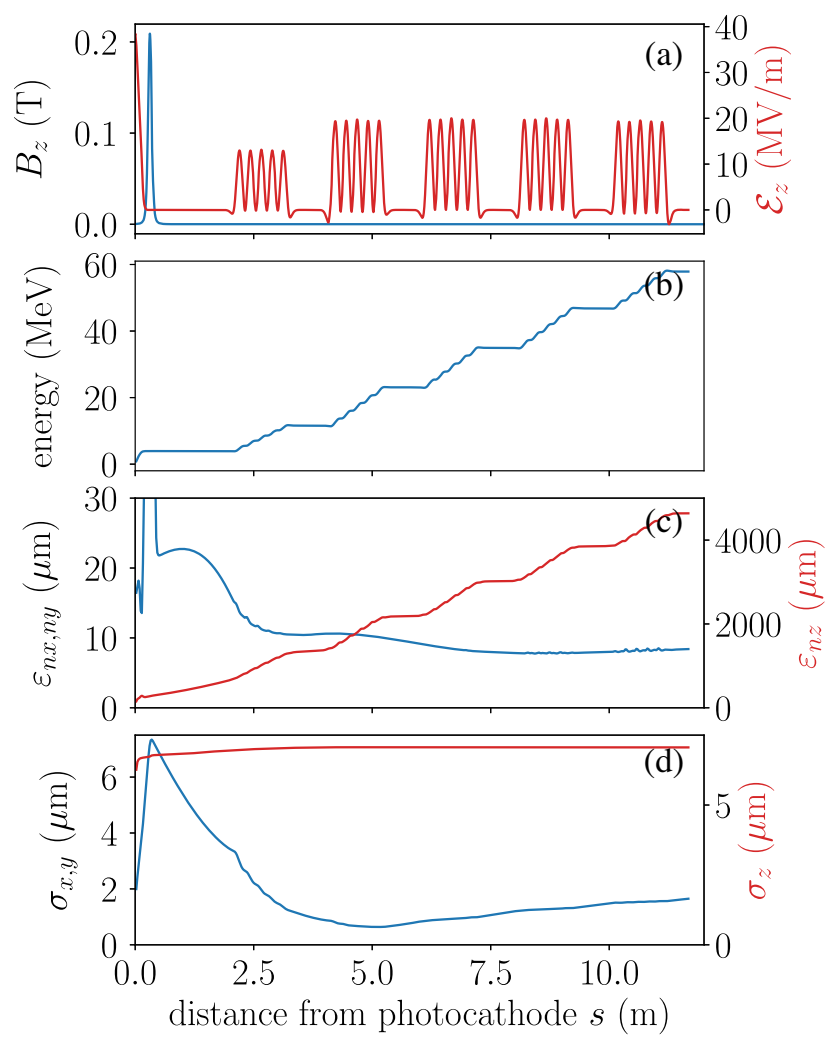

FIG. 7. Axial electric $\mathcal{E}_{z}$ (red trace) and magnetic $B_{z}$ (blue trace) fields experienced by the reference particle as it propagates along the optimized photoinjector (a) with corresponding kinetic energy (b), transverse (blue) and longitudinal (red) beam emittances (c), and sizes (d) evolving along the injector.

the microbunching instabilities. Consequently, a laser heater is required to increase the uncorrelated energy spread.

The correspondingly revised diagram of the accelerator beam line shown in Fig. 8 was used as a new starting point to investigate the performance of the proposed bunchshaping process with ELEGANT tracking simulations taking into account the transverse beam dynamics.

Another challenge associated with the bunch formation pertains to the temporal resolution of the bunch shaping process. Ultimately, the laser pulse shape can only be controlled on a timescale $\delta t \geq 1 /\left(2 \pi \delta f_{L}\right)$ limited by the bandwidth of the photocathode laser $\delta f_{L}$. Contemporary laser systems are capable to $\delta t \leq 150 \mathrm{fs}$ (rms) [60]. Additionally, the electron bunch shape is also affected by the time response of the photoemission process. Given the required charge of $\sim 10 \mathrm{nC}$, we consider a $\mathrm{Cs}_{2} \mathrm{Te}$ photocathode with temporal response numerically

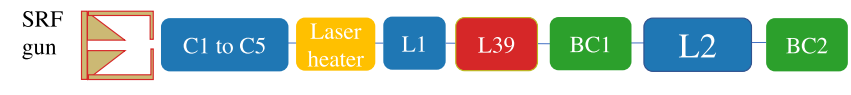

FIG. 8. Updated accelerator design, with the addition of the injector beam line and a laser heater section. 
investigated in Refs. [61,62]. Recent measurements confirm that $\mathrm{Cs}_{2} \mathrm{Te}$ has a photoemission response time below 370 fs [63]. Figure 5 compares the optimized ideal laser pulse shape described by Eq. (8) with the cases when the photocathode response time and the laser finite bandwidth are taken into account. The added effects have an insignificant impact on the final distribution due to relatively slow temporal variations in the required current distribution.

\section{FINAL ACCELERATOR DESIGN}

The conceptual design developed so far with the help of 1D simulations provides guidance to a complete design of the accelerator beam line. In the following sections, we detail a possible accelerator beam line configurationincluding realistic beam line components for the linac and bunch-compressing sections - to verify the transverse and longitudinal beam-dynamics performances via start-to-end tracking simulations.

\section{A. Accelerator components}

Linacs.-For the $650 \mathrm{MHz}$ L1 and L2 SRF linacs we adopted cryomodules proposed for the PIP-II project [64]. The linac L1 consists of two cryomodules, and L2 has eight cryomodules. Each cryomodule includes six cavities containing five cells. We assume that in $\mathrm{cw}$ operation each cavity provides up to $20 \mathrm{MV} / \mathrm{m}$ average accelerating gradients. The quadrupole magnet doublets are located between cryomodules and produce a pseudoperiodic oscillation of the betatron functions. The two cavities used in the 3.9 GHz L39 SRF linac are similar to the cavity described in Ref. [49].

Bunch compressors.-We use an arc-shaped bunch compressor consisting of a series of FODO cells, where each cell contains two quadrupoles and two dipole magnets [65]. This configuration provides a positive $R_{56}$ [66-69]

$$
R_{56} \simeq \frac{\theta_{\text {total }}^{2} L_{\text {total }}}{4 N_{\text {cell }}^{2} \sin ^{2}\left(\psi_{x} / 2\right)},
$$

where $\theta_{\text {total }}$ is the total bending angle, $L_{\text {total }}$ is the total path length, $N_{\text {cell }}$ is the total number of FODO cells, and $\psi_{x}$ is the horizontal phase advance per cell. The dipole magnet bending angles can be used to tune the $R_{56}$. The bending angle or dipole polarity from cell to cell does not need to be identical, but the number of cells should be selected to realize a phase advance $\psi_{x \text {,total }}=2 n \pi$ (with $n$ integer) over the compressor to achieve the first-order achromat.

The second-order longitudinal dispersion produced by the bunch compressor is given by [70,71]

$$
T_{566}=\int_{0}^{L}\left[\frac{\eta_{1, x}\left(s^{\prime}\right)}{\rho\left(s^{\prime}\right)}+\frac{\eta_{x}^{\prime 2}\left(s^{\prime}\right)}{2}\right] \mathrm{d} s^{\prime}
$$

where $L$ is the length of the beam line, $\rho$ is the bending radius, $\eta_{1, x}(s) \equiv\left(E_{0}{ }^{2} / 2\right) \partial^{2} x(s) / \partial E^{2}$ is the second-order horizontal dispersion function, and $\eta_{x}^{\prime}(s)$ is the derivative of the dispersion function. We incorporate 12 sextupole magnets to control the $T_{566}$ and 12 octupole magnets to cancel the third-order longitudinal transfer-map element $U_{5666}$ computed over BC1. If needed, a nonvanishing value of $U_{5666}$ can enable higher-order control over the LPS correlation [51,72].

The sextupole and octupole magnets are also used to zero the chromatic transfer-map elements $T_{166}, T_{266}$, and $U_{1666}$, resulting in the bunch compressors being achromatic up to the third order.

Figure 9 displays the $\mathrm{BC} 1$ configuration along with the evolution of the betatron functions and relevant horizontal chromatic $\left(\eta_{x}, \eta_{1, x}\right.$, and $\left.\eta_{2, x}\right)$ and longitudinal accumulated transfer-map elements $\left(R_{56}^{0 \rightarrow s}, T_{566}^{0 \rightarrow s}\right.$, and $\left.U_{5666}^{0 \rightarrow s}\right)$ up to third order as a function of the beam line coordinate $s$. It has two arcs, one bending the beam trajectory by $22.92^{\circ}$ and another one bending it back. Each bending magnet has

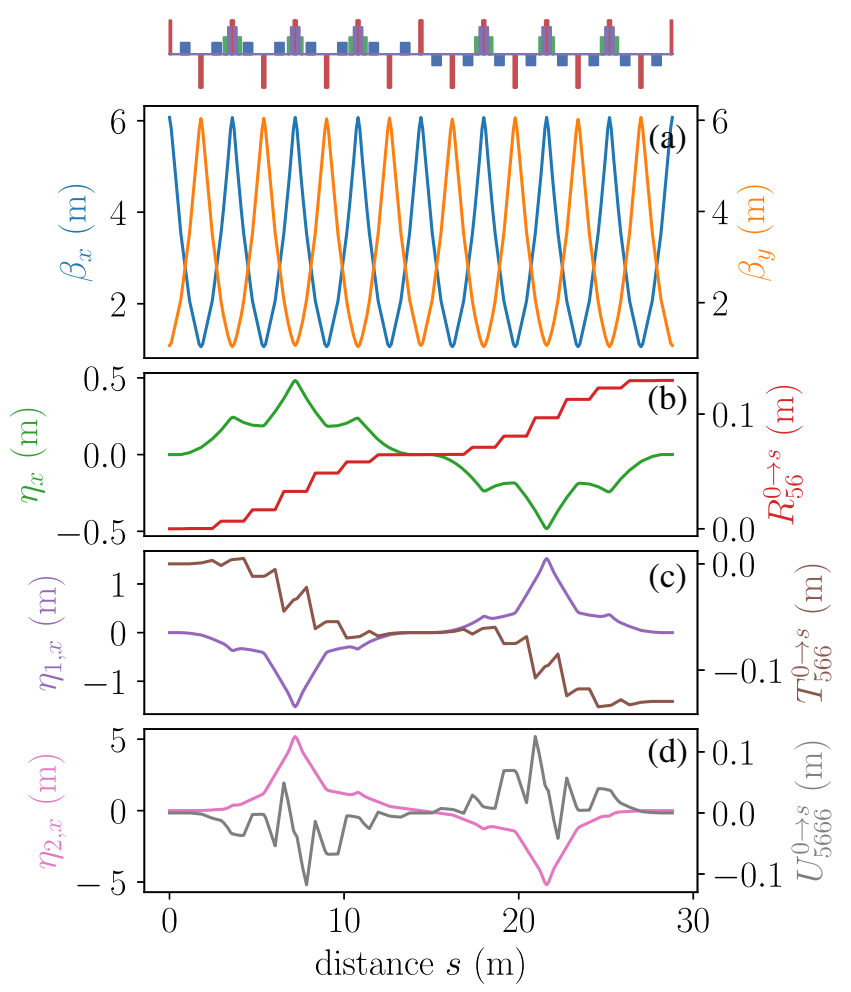

FIG. 9. Layout of bunch compressor BC1 (top diagram) with evolution of associated betatron function (a) and pertinent linear (b), second-order (c), and third-order (d) transfer-map elements along the beam line (with $s=0$ corresponding to the beginning of $\mathrm{BC} 1$ ). In plots (b)-(d) the left and right axes refer to the horizontal chromatic functions $\eta_{i, x}$ and accumulated longitudinal transfer-map elements from 0 to location $s$ along BC1. In the top diagram the red, blue, green, and purple rectangles correspond, respectively, to quadrupole, dipole, sextupole, and octupole magnets. 
the bending angle $\theta=2.865^{\circ}$. This design relaxes the requirement on the sextupole- and octupole-magnet strengths necessary to produce a $T_{566}<0$ (see Table II) while simultaneously achieving a third-order achromat. Likewise, the reversed-arc configuration is also beneficial to mitigate CSR-induced emittance growth as discussed in Ref. [73]. The strengths of the sextupole magnets were optimized using ELEGANT to achieve the required $T_{566}$ across $\mathrm{BC} 1$ while obtaining a second-order achromat by constraining $T_{166}=T_{266}=0$. The three pairs of sextupole magnets in the second arc are mirror symmetric to the first three pairs, with opposite-polarity magnet strengths. During the design process, the first pair of sextupole magnets was inserted close to the region of the first arc with the highest dispersion for tuning the desired $T_{566}$; its mirror symmetry pair was placed in the second arc and separated by $2 \pi$ phase advance. Another two pairs of sextupole magnets were subsequently inserted for tuning $T_{166}$. Similarly, their mirror symmetry pairs were separated by $2 \pi$ phase advance. Finally, six pairs of octupole magnets were inserted to zero the overall $U_{i 666} i=1,2,5$ transfermap elements, where the same design process was employed. The $\mathrm{BC} 2$ compressor requires both $R_{56}$ and $T_{566}$ to be positive, which is naturally provided by the arc bunch compressor introduced earlier. It has a total bending angle of $32.63^{\circ}$, and each dipole has a bending angle of $4.079^{\circ}$. Similar to BC1, we used sextupole- and octupolemagnet families to adjust both $T_{566}$ and $U_{5666}$ and produce the third-order achromat. The BC2 lattice appears in Fig. 10 along with the evolution of the betatron functions and relevant chromatic elements. Finally, the layout of the two bunch compressors is presented in Fig. 11.

Matching sections.-All accelerator components are connected using matching sections composed of quadrupole magnets and drift spaces.

The evolution of the betatron functions from the injector exit up to the end of BC2 appears in Fig. 12. Throughout the entire accelerator, the betatron functions are maintained to values $\beta_{x, y}<30 \mathrm{~m}$.

\section{B. Tracking and optimization}

The beam distribution obtained at the exit of the injector from ASTRA was used as input to ELEGANT for tracking and optimization. We found that we need to increase the slice energy spread to $\sim 75 \mathrm{keV}$ using the laser heater to suppress the microbunching instability [74,75]. However, in this study, we numerically added random noise with Gaussian distribution to the macroparticles' energy using the SCATTER element available in ELEGANT. Thus, Fig. 13 shows the actual LPS distribution used at the beginning of the accelerator in tracking studies.

The accelerator settings obtained with TwICE were used as a starting point in the accelerator optimization including transverse effects. The fine-tuning of the above-described accelerator components was accomplished using ELEGANT.

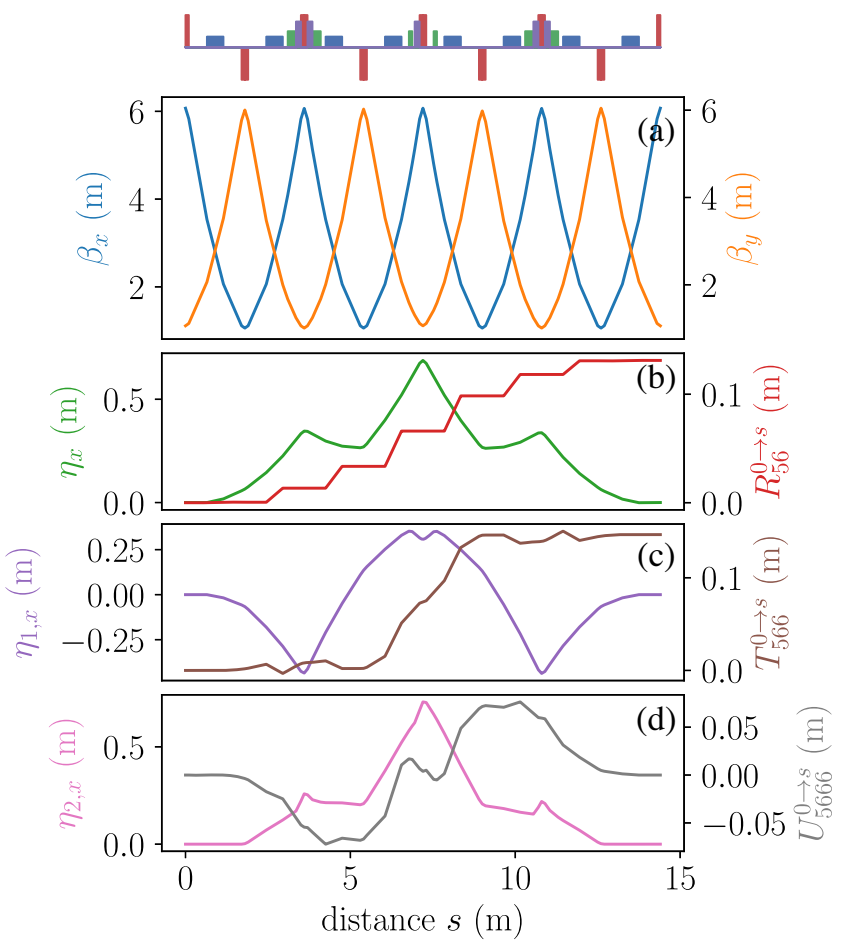

FIG. 10. Layout of bunch compressor BC2 (top diagram) with evolution of associated betatron function (a) and pertinent linear (b), second-order (c), and third-order (d) transfer-map elements along the beam line (with $s=0$ corresponding to the beginning of $\mathrm{BC} 2$ ). In plots (b)-(d) the left and right axes refer to the horizontal chromatic functions $\eta_{i, x}$ and accumulated longitudinal transfer-map elements from 0 to location $s$ along BC2. The top diagram follows the same conventions as in Fig. 9.

A multiobjective optimization was applied to determine the twelve accelerator parameters controlling the longitudinal dynamics, i.e., voltages and phases of L1, L2, L39, and values of $R_{56}, T_{566}$ in two bunch compressors.
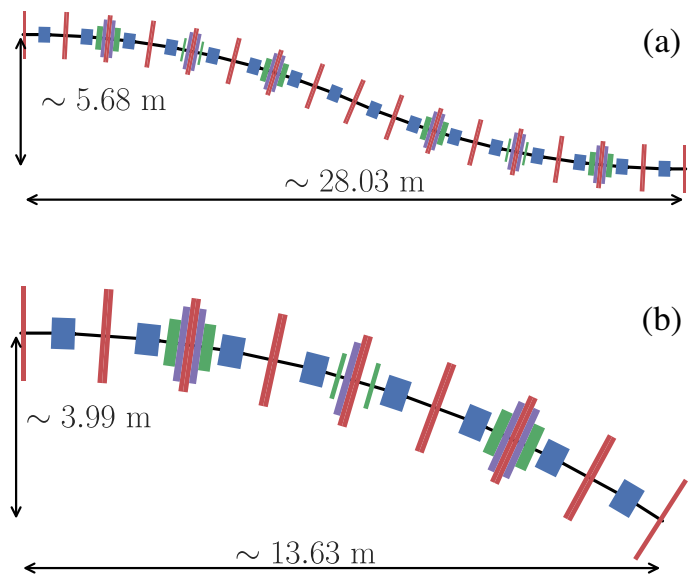

FIG. 11. The geometry of the bunch compressors BC1 (a) and BC2 (b), where red, blue, green, and purple rectangles are quadrupoles, dipoles, sextupoles, and octupoles magnets, respectively. 


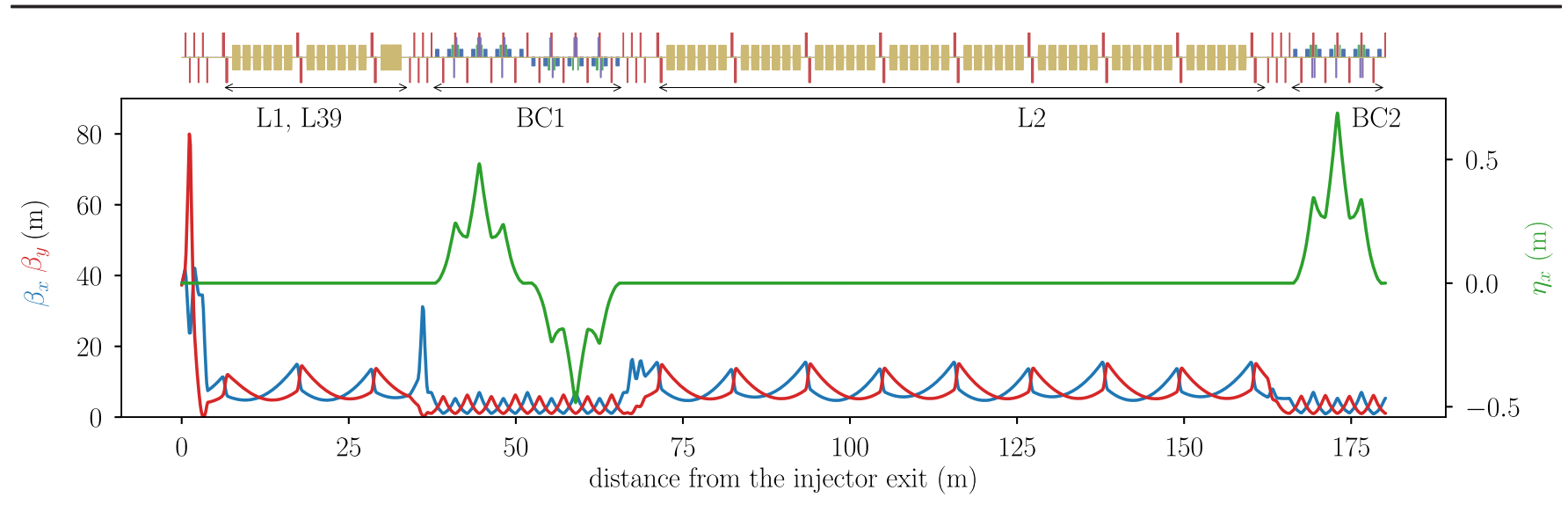

FIG. 12. Evolution of the betatron (left axis) and horizontal dispersion (right axis) functions along the proposed linac. The vertical dispersion is zero throughout the linac. The magnetic-lattice color coding for the element follows Fig. 9 with the accelerating cavities shown as gold rectangles.
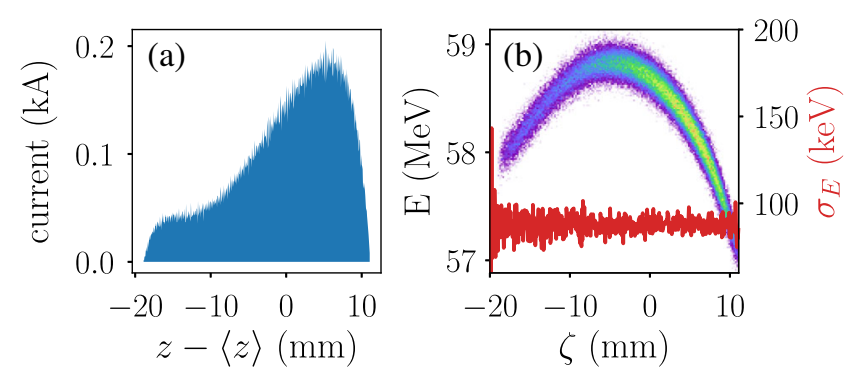

FIG. 13. Current profile (a) and associated LPS (b) distributions simulated with ASTRA at the end of the photoinjector (see Fig. 6) with added uncorrelated fraction energy spread following a Gaussian distribution with rms spread $\sigma_{E} / E=1.5 \times 10^{-3}$. In plot (b) the red trace represents the slice rms energy spread $\sigma_{E}$.

The resulting beam distribution obtained downstream of $\mathrm{BC} 2$ was then used to compute the wakefield generated in a $180 \mathrm{GHz}$ corrugated waveguide considered for the role of the wakefield accelerator in [43]. The resulting peak accelerating field and transformer ratio were then adopted as objective functions to be maximized with the accelerator parameters as control variables. The trade-off between peak accelerating field and transformer ratio was quantified in Eq. (30) of Ref. [10], hence providing a good measure to verify whether our optimization reaches the optimal Pareto front. The optimal accelerator settings and final beam parameters are summarized in Table IV. The LPS distribution at the end of the accelerator is shown in Fig. 14. We also calculated that the $\sim 1 \mathrm{GeV}, 10 \mathrm{nC}$ electron bunch having this distribution produces a peak wakefield of $94.26 \mathrm{MV} / \mathrm{m}$ with a transformer ratio of 5 propagating in a corrugated waveguide. Figure 15 demonstrates that our optimization has reached the optimal set of solutions, where the Pareto front closely follows the analytically calculated tradeoff curve [10]. The obtained current profile produces a wakefield amplitude $\sim 15 \%$ lower than the one expected from the ideal distribution for a transformer ratio
$\mathcal{R} \simeq 5$. Such an agreement gives confidence in our optimization approach based on the trade-off between peak accelerating field and transformer ratio. The simulations also indicate that the horizontal transverse emittance increases to $\varepsilon_{n x}=31 \mu \mathrm{m}$ due to the CSR and chromatic aberrations in the electron bunch having large correlated energy variations. Although significant, this emittance dilution is still acceptable.

Our main result is shown in Fig. 16. It compares the final distribution and wakefield with that of the target distribution and wakefield from Fig. 2. A good agreement

TABLE IV. Main accelerator parameters and beam parameters at the end of $\mathrm{BC} 2$.

\begin{tabular}{lcc}
\hline \hline Parameter & Value & Unit \\
\hline Accelerating voltage L1 & 193.22 & $\mathrm{MV}$ \\
Phase L1 & 21.64 & $\mathrm{deg}$ \\
Frequency L1 & 650 & $\mathrm{MHz}$ \\
Accelerating voltage L39 & 9.73 & $\mathrm{MV}$ \\
Phase L39 & 202.52 & $\mathrm{deg}$ \\
Frequency L39 & 3.9 & $\mathrm{GHz}$ \\
$R_{56}$ for bunch compressor 1 (BC1) & 0.1294 & $\mathrm{~m}$ \\
$T_{566}$ for bunch compressor 1 (BC1) & -0.1294 & $\mathrm{~m}$ \\
$U_{5666}$ for bunch compressor 1 (BC1) & 0 & $\mathrm{~m}$ \\
Accelerating voltage L2 & 857.92 & $\mathrm{MV}$ \\
Phase L2 & 26.05 & $\mathrm{deg}$ \\
Frequency L2 & 650 & $\mathrm{MHz}$ \\
$R_{56}$ for bunch compressor 2 $(\mathrm{BC} 2)$ & 0.1312 & $\mathrm{~m}$ \\
$T_{566}$ for bunch compressor 2 $(\mathrm{BC} 2)$ & 0.1465 & $\mathrm{~m}$ \\
$U_{5666}$ for bunch compressor $2(\mathrm{BC} 1)$ & 0 & $\mathrm{~m}$ \\
Final beam energy & 998 & $\mathrm{MeV}$ \\
Final beam bunch length & 414 & $\mu \mathrm{m}$ \\
Final beam normalized emittance, $\varepsilon_{n x}$ & 31 & $\mu \mathrm{m}$ \\
Final beam normalized emittance, $\varepsilon_{n y}$ & 12 & $\mu \mathrm{m}$ \\
Peak accelerating wakefield $\left|\mathcal{E}_{+}\right|$ & 94.3 & $\mathrm{MV} / \mathrm{m}$ \\
Peak decelerating wakefield $\left|\mathcal{E}_{-}\right|$ & 18.8 & $\mathrm{MV} / \mathrm{m}$ \\
Transformer ratio $\mathcal{R}$ & 5.0 & $\cdots$ \\
\hline \hline
\end{tabular}



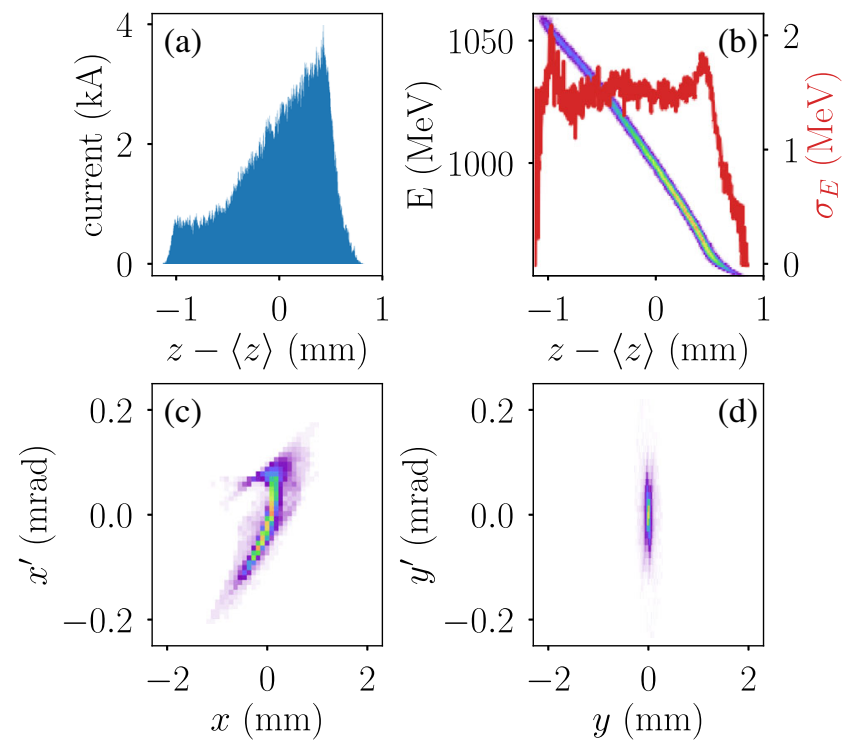

FIG. 14. Current (a) with associated LPS (b), and transverse horizontal (c) and vertical (d) phase-space distributions simulated with ELEGANT at the end of BC2 using the optimized linac and bunch-compressor settings summarized in Table IV and the injector distributions from Fig. 13. In plot (b) the red trace represents the slice rms energy spread $\sigma_{E}$.

manifests that, indeed, the drive electron bunch with a highly asymmetric current profile can be obtained without employing the collimators.

A comparison of Tables III and IV indicates that the final accelerator settings optimized by ELEGANT deviate less than $10 \%$ from those obtained with TWICE. It justifies the strategy taken in this study to solve the difficult problem of formation of temporally shaped electron bunches for a beam-driven collinear wakefield accelerator in two steps.

The nonlinear correlation observed in the tail of the LPS distribution downstream of BC2 [see Fig. 14(b)] originates from the CSR. As the beam is compressed inside the bunch

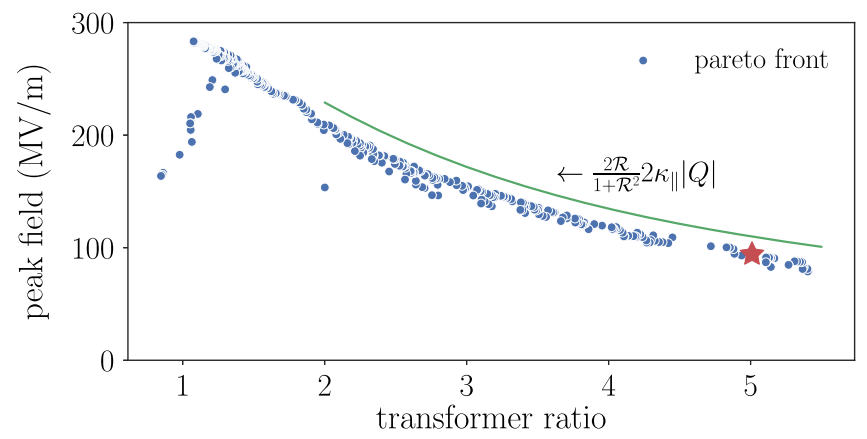

FIG. 15. Comparison of the Pareto front with the analytical trade-off curve between the peak field and transformer ratio described by Eq. (30) of Ref. [10]. Each blue dot represents a numerically simulated configuration with the red star representing the configuration with parameters listed in Table IV.

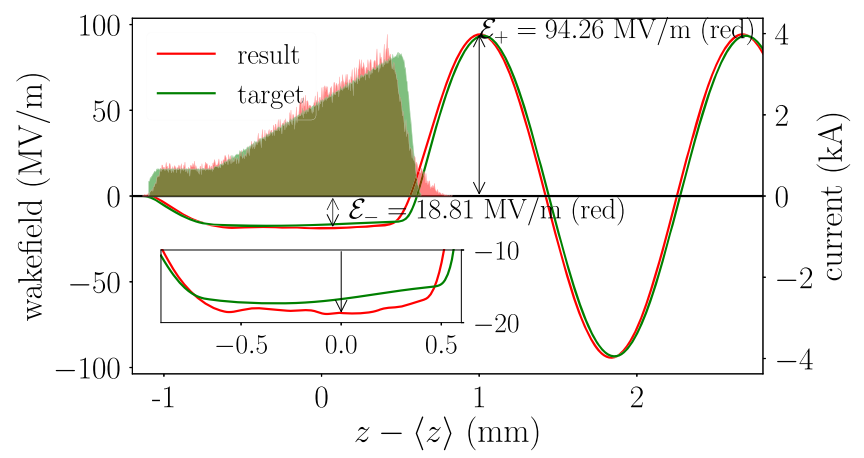

FIG. 16. Target (from Fig. 2) and optimized final current distributions (respectively shown as green- and red-shaded curves) with associated wakefields (respectively displayed as green and red traces). The transformer ratio for the simulated distribution is $\mathcal{R}=5.0$.

compressors, its tail experiences a stronger CSR force due to its peak current being higher than the rest of the bunch. It is worth noting that ELEGANT uses a 1D projected model to treat the CSR effect. The applicability of such a 1D treatment is conditioned by the Derbenev's criterion [76], which suggests that projecting the bunch distribution onto a line-charge distribution may overestimate the CSR force, particularly when the bunch has a large transverse-to-longitudinal aspect ratio $\mathcal{A}(s) \equiv\left[\sigma_{x}(s) / \sigma_{z}(s)\right] \sqrt{\left[\sigma_{x}(s) / \rho(s)\right]}$. In our design, the condition $\mathcal{A} \ll 1$ was not rigorously followed (but rather the softer condition $\mathcal{A}<1$ was achieved), suggesting that the impact of CSR may be overestimated in some regions of the bunch compressors.
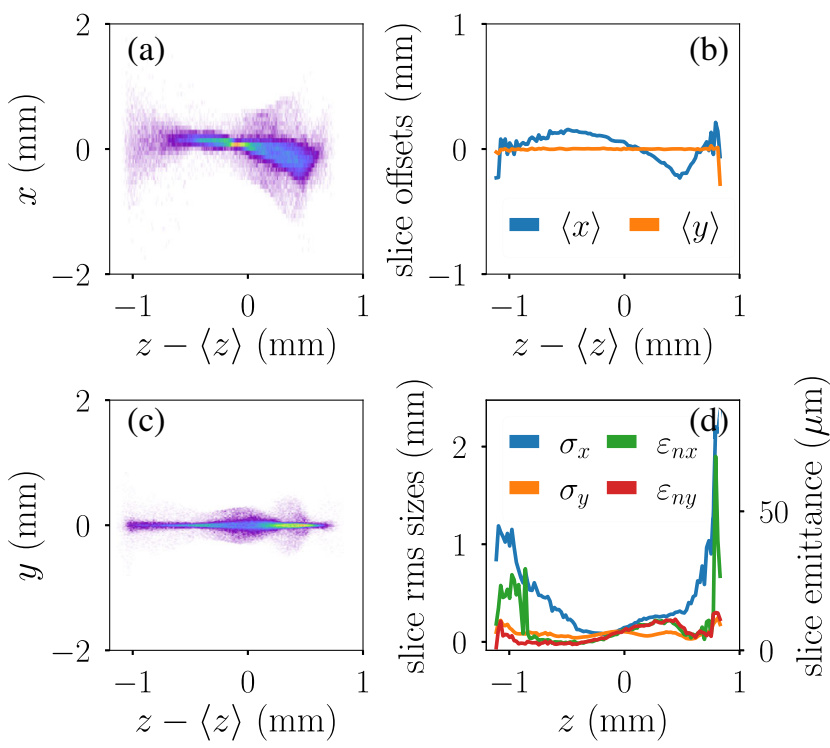

FIG. 17. Final $(z, x)$ (a) and $(z, y)$ (c) beam distributions corresponding to the data shown in Fig. 14, and slice analysis for positions $\langle x\rangle$ and $\langle y\rangle$ (b) and rms beam size and emittances $(d)$. 
We also note that the final beam distribution exhibits significant longitudinal-horizontal $z-x$ correlations due to CSR effects; see Fig. 17. Although the associated projected-emittance dilution is tolerable, the electrons in the longitudinal slices with the horizontal offsets seen in Fig. 17(c) will excite transverse wakefields in the CWA and ultimately seed the BBU instability. These offsets come from CSR-induced energy loss occurring in the $\mathrm{BC} 2$ that breaks the achromatic property of this beam line. Understanding the impact of this distribution feature in the CWA linac along with finding mitigation techniques is a current research focus.

\section{Impact of jitter on performances}

In order to validate the robustness of the proposed design, it is instructive to investigate the sensitivity of the proposed shaping technique to shot-to-shot jitters of the amplitude and phase of the accelerating field in the linac's structures. Consistent with LCLS-II specifications [77], we considered the relative rms amplitude jitter of $0.01 \%$ and the phase jitter of 0.01 degree. For simplicity, we assume that the injector produced identical bunches, as shown in Fig. 6, and performed 100 simulations of the accelerator beam line (from the injector exit to the exit of $\mathrm{BC} 2$ ) for different random realizations of the phase and amplitude for linacs L1, L2, and L39. The errors in linac settings were randomly generated using a Gaussian probability function with standard deviations of $0.01 \%$ and $0.01^{\circ}$. Figure 18 presents the wakefield averaged over the 100 simulations and indicates that a stable transformer ratio $5.00 \pm 0.05$ can be maintained owing to the stable beam produced in the superconducting linac.

Likewise, we investigated the impact of magnetic-field jitter for all magnets forming the lattice by considering an

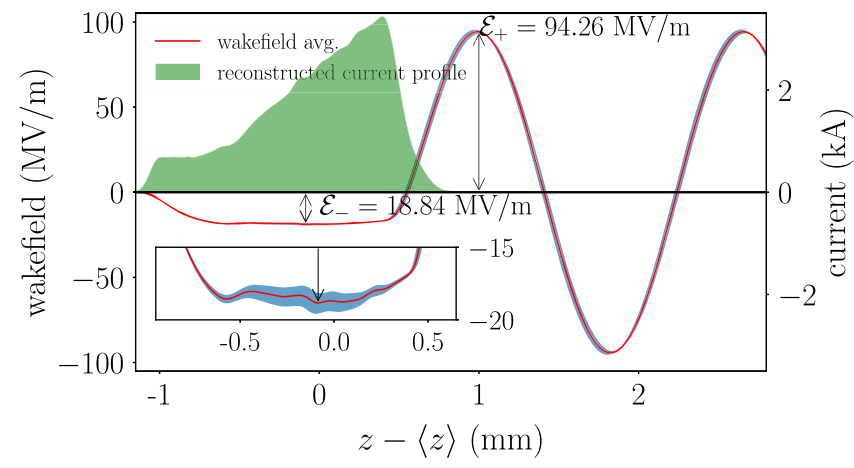

FIG. 18. Wakefields obtained from 100 simulations with jitter in linacs L1, L2, and L39. All cavities are taken to have relative jitter in accelerating voltage of $0.01 \%$ and phase jitter $0.01^{\circ}$. The red line shows the average wakefield while the blue shaded region represents the fluctuation of wakefields due to jitter over 100 random realizations of the linac settings. The average transformer ratio is $5.00 \pm 0.05$. The reconstructed current profile (greenshaded curve) is obtained numerically using Eq. (4).

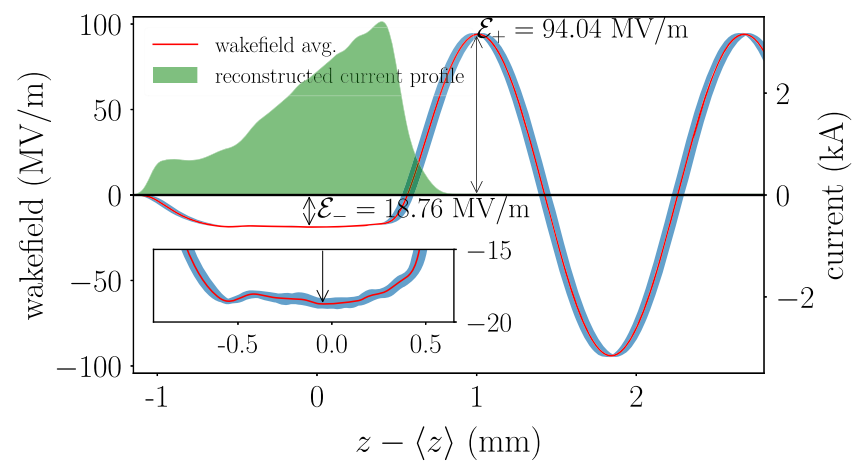

FIG. 19. Wakefields obtained from 100 simulations with factional magnetic field jitter $\Delta B / B=10^{-4}$ on all the magnets. The red line shows the average wakefield while the blue shaded region represents the fluctuation of wakefields due to jitter over 100 random realizations. The reconstructed current profile (greenshaded curve) is obtained numerically using Eq. (4).

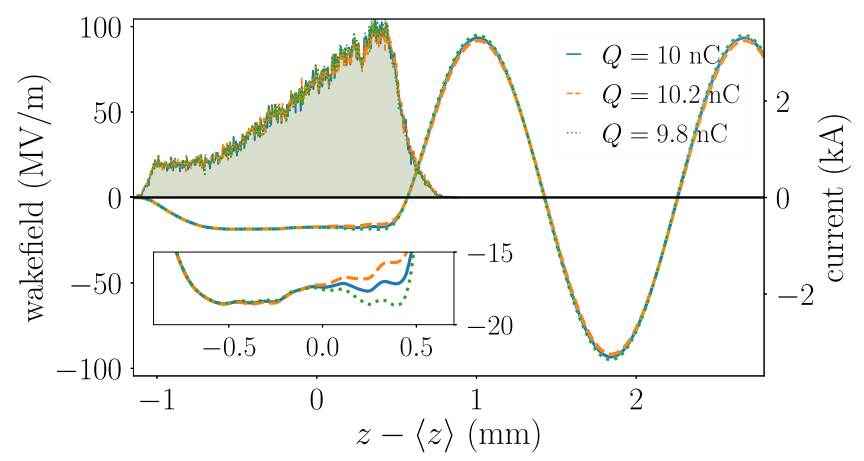

FIG. 20. Current distribution (shaded curves, right axis) and associated wakefields (traces) for the nominal charge and $\pm 2 \%$ relative change in charge (9.8 and $10.2 \mathrm{nC}$ ).

rms fractional field jitter of $\frac{\Delta B}{B}=10^{-4}$ corresponding to fast-time-scale current stability (current "ripple") typically attained in high-precision power supplies encountered in accelerators. Figure 19 presents the current distribution with the produced wakefield and confirms the minimal impact of the field jitter on the shaping performances: the transformer ratio computed over the 100 random realizations is $\mathcal{R}=5.01 \pm 0.03$.

Finally, we observe the impact of charge fluctuation on the shaping to be tolerable. Cathode-to-end simulations combining ASTRA and ELEGANT indicate that a relative charge variation of $+2 \%$ (respectively $-2 \%$ ) yields a relative change in the transformer ratio of $-2 \%$ (respectively $+1 \%$ ) and a relative variation in peak field of $-1.7 \%$ (respectively $+1.7 \%$ ); see Fig. 20 .

\section{SUMMARY}

We have presented the design of an accelerator capable of generating $1 \mathrm{GeV}$ electron bunches with a highly asymmetric current profile and a large energy chirp 
required for a collinear wakefield accelerator. It has been achieved without the use of collimators. Our approach is based on $a b$ initio temporal shaping of the photocathode laser pulse followed by nonlinear manipulations of the electron distribution in the longitudinal phase space throughout the accelerator using collective effects and precision control of the longitudinal dispersion in two bunch compressors up to the third order. Finding the optimal design consisted of first implementing a simplified accelerator model and using it for backward tracking of the longitudinal phase space distribution of electrons through the main accelerator to provide the longitudinal phase space distribution required from the injector. The program TwICE was developed to support such a capability and used to optimize the global linac parameters and time-of-flight properties of bunch compressors. Second, the simulation of the photoinjector using ASTRA was performed to generate the required distribution. Third, the linac design was refined using ELEGANT to account for the transverse beam dynamics. Finally, formation of longitudinally shaped drive bunches capable of producing in the collinear wakefield accelerator a transformer ratio of $\sim 5$ and a peak accelerating wakefield close to $100 \mathrm{MV} / \mathrm{m}$ has been numerically demonstrated.

Although the proposed accelerator design is promising, we note that further work is required to investigate whether the same accelerator can accelerate the low-charge, lowemittance "witness bunches" that would be accelerated to multi-GeV energies in the collinear wakefield accelerator and used for the generation of $\mathrm{X}$ rays in the downstream free-electron laser. Discussion of this research is the subject of a forthcoming publication.

\section{ACKNOWLEDGMENTS}

The authors are grateful to Dr. Stanislav Baturin (NIU) for useful discussions. W. H. T. thanks Y. Park (UCLA) for several discussions on simulation studies. This work is supported by the U.S. Department of Energy, Office of Science, under Award No. DE-SC0018656 with Northern Illinois University and Contract No. DE-AC0206CH11357 with Argonne National Laboratory.

\section{APPENDIX A: ONE-DIMENSIONAL TRACKING MODEL}

A simple one-dimensional tracking program TWICE [47] was developed for rapid assessment of the longitudinal dynamics of electrons in linear accelerators. The program adopts an approach similar to the one used in LiTrack [48], where only the accelerator components affecting the longitudinal beam dynamics are considered and modeled analytically. A detailed description of TWICE is published in [47]. In brief, the beam is represented by a set of $N$ macroparticles with identical charges $Q / N$ and given a set of initial LPS coordinates $\left(z_{i}, E_{i}\right)$. A transformation $\left(z_{f}, E_{f}\right)=f\left(z_{i}, E_{i}\right)$ is applied to obtain final coordinates in the LPS.

\section{Single particle dynamics}

In TWICE the transformation for a macroparticle with coordinates $\left(z_{i}, E_{i}\right)$ passing through a radio-frequency (rf) linac is given by

$$
\left(\begin{array}{c}
z_{f} \\
E_{f}
\end{array}\right)=\left(\begin{array}{c}
z_{i} \\
E_{i}\left(z_{i}\right) \pm e V \cos \left(k z_{i}+\varphi\right)
\end{array}\right),
$$

where $V, k$, and $\varphi$ are, respectively, the accelerating voltage, wave-vector amplitude, and off-crest phase associated with the accelerating section, and $e$ is the electronic charge. In the latter and following equations the \pm sign indicates the forward (+) and backward (-) tracking process detailed in Appendix A 3. Similarly, the transformation through a longitudinally dispersive section, such as a bunch compressor, is given by

$$
\left(\begin{array}{c}
z_{f} \\
E_{f}
\end{array}\right)=\left(\begin{array}{c}
z_{i} \pm\left[R_{56} \frac{E_{i}-E_{0}}{E_{0}}+T_{566}\left(\frac{E_{i}-E_{0}}{E_{0}}\right)^{2}\right] \\
E_{i}
\end{array}\right),
$$

where $E_{0}$ is the reference-particle energy assumed to remain constant during the transformation, and $R_{56} \equiv$ $E_{0} \frac{\partial z_{f}}{\partial E_{i}}$ and $T_{566} \equiv \frac{E_{0}^{2}}{2} \frac{\partial^{2} z_{f}}{\partial E_{i}^{2}}$ are the first- and second-order longitudinal-dispersion functions introduced by the beam line. It should be noted that, given our LPS coordinate conventions, a conventional four-bend "chicane" magnetic bunch compressor has a longitudinal dispersion $R_{56}>0$. The latter equation ignores energy loss, e.g., due to incoherent synchrotron radiation, occurring in the beam line magnets.

\section{Collective effects}

In TWICE, we implemented collective effects as an energy kick approximation using the transformation

$$
\left(\begin{array}{c}
z_{f} \\
E_{f}
\end{array}\right)=\left(\begin{array}{c}
z_{i} \\
E_{i}\left(z_{i}\right) \pm \Delta E\left(z_{i}\right)
\end{array}\right)
$$

where $\Delta E(z)$ represents the energy change associated with the considered collective effect. The treatment of collective effects is modeled as a $z$-dependent energy kick $\Delta E(z)$ taken downstream of beam line elements as specified for the forward and backward tracking with the diagram shown in Fig. 21. The implemented collective effects include wakefields modeled after a user-supplied Green's function, one-dimensional steady-state coherent synchrotron radiation (CSR), and longitudinal space charge (LSC) described via an impedance. The collective effects require the estimation of the beam's charge density, which is done in TWICE either using a standard histogram binning method 


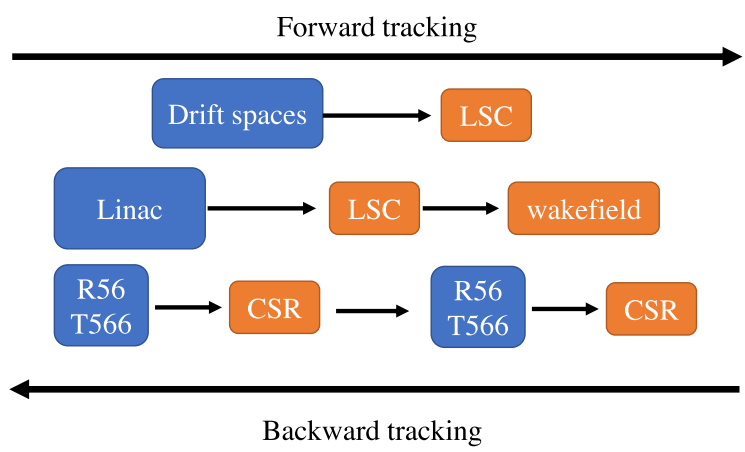

FIG. 21. Treatment of collective effects as energy kicks downstream of beam line elements. In forward (respectively backward) tracking, transformations of beam line elements (respectively energy kicks) were applied, followed by energy kicks (respectively beam line elements).

with noise filtering or via the kernel-density estimation technique [78].

In order to model the impact of a wakefield, the charge distribution $q(z)$ is directly used to compute the wake potential given a tabulated Green's function

$$
W(z)=\int_{0}^{z} q\left(z^{\prime}\right) G\left(z-z^{\prime}\right) \mathrm{d} z^{\prime}
$$

The change in energy is computed as $\Delta E(z)=L W(z)$, where $L$ is the effective length where the beam experiences the wakefield.

The LSC is implemented using a one-dimensional model detailed in [79], where the impedance per unit length is

$$
Z(k)=i \frac{Z_{0}}{\pi \gamma r_{b}} \frac{1-2 I_{1}\left(\xi_{b}\right) K_{1}\left(\xi_{b}\right)}{\xi_{b}},
$$

where $\xi_{b} \equiv k r_{b} / \gamma ; I_{1}$ and $K_{1}$ are modified Bessel functions of the first and second kind, respectively; and $k, Z_{0}$ and $r_{b}$ are, respectively, the wave-vector amplitude, impedance of free space and a user-defined transverse beam radius, and $\gamma$ is the Lorentz factor. Given the charge density, the Fourier-transformed current density $\tilde{I}(k)$ is derived from

$$
\tilde{I}(k)=\mathcal{F}[c q(z)],
$$

with $\mathcal{F}$ representing the Fourier transform. The change in energy is computed as

$$
\Delta E=-\mathcal{F}^{-1}[e Z(k) \tilde{I}(k) L],
$$

where $\mathcal{F}^{-1}$ is the inverse Fourier transform, and $L$ is the effective distance along which the LSC interaction occurs. In order to account for LSC during acceleration, $\gamma$ is replaced by the geometry average $\sqrt{\gamma_{i} \gamma_{f}}$ of the Lorentz factors computed at the entrance $\gamma_{i}$ and exit $\gamma_{f}$ of the linac section.

Finally, CSR energy kicks are applied downstream of the dispersive beam line elements. For instance, a CSR energy kick can be applied after a dispersive element with userdefined length and angle described by $R_{56}$ and $T_{566}$. The effect of CSR is described using a one-dimensional model commonly implemented in other beam-dynamics programs [80]. To simplify the calculation, only the steady-state CSR is considered in TWICE. The energy loss associated with CSR is obtained from $[81]^{2}$

$$
\Delta E(z)=\rho \theta \frac{\mathrm{d} E}{\mathrm{~d} c t}=-\theta \frac{\gamma m_{e} c^{2} r_{e}}{e} \int_{-\infty}^{z} \frac{\partial q\left(z^{\prime}\right)}{\partial z^{\prime}} I_{c s r}\left(z, z^{\prime}\right) \mathrm{d} z^{\prime},
$$

with the integral kernel defined as

$$
I_{c s r}\left(z, z^{\prime}\right)=\frac{4 u\left(u^{2}+8\right)}{\left(u^{2}+4\right)\left(u^{2}+12\right)},
$$

where $\theta$ is the angle, $m_{e} c^{2}$ is the electron rest mass energy, $r_{e}$ is the classical electron radius and the variable $u$ is the solution of $\frac{\gamma^{3}\left(z-z^{\prime}\right)}{\rho}=\frac{u^{3}}{24}+\frac{u}{2}$. CSR introduces an energy loss strongly dependent on the bunch length, which varies within the dispersive sections used to compress the bunch. Consequently, the longitudinally dispersive beam lines are segmented into several elements with individual $\left(R_{56}, T_{566}\right)$ parameters. A CSR kick is applied after each of the elements. A conventional chicane-type bunch compressor is usually broken into two sections (two mirror-symmetric doglegs) but can in principle be divided into an arbitrary number of segments to improve the resolution at the expense of computational time.

\section{Backward tracking}

An important feature of TWICE is its capability to track the beam in the forward or backward directions [indicated by the \pm sign in Eqs. (A2) and (A3)] in the presence of collective effects (so far LSC, CSR, and wakefield effects are included). The effects of LSC and wakefield are straightforward to implement as they only involve a change in energy, while handling of the CSR requires extra care since the particles' positions also change throughout the dispersive section. Therefore, an energy kick is applied after the beam line element in the forward-tracking mode and before the beam line element in backward-tracking mode, as shown in Fig. 21. Although the treatment of CSR is not exact, it nonetheless provides a good starting point to account for the effect.

\footnotetext{
${ }^{2}$ The variable $z$ here for CSR calculation refers to the relative position from the bunch centroid with bunch head at $z>0$.
} 
[1] G. Voss and T. Weiland, The wakefield acceleration mechanism, Technical Report No. DESY-82-074, DESY, 1982.

[2] R. J. Briggs, T. J. Fessenden, and V. K. Neil, Electron autoacceleration, in Proceedings of the 9th International Conference on the High-Energy Accelerators, Stanford, California, USA (1974), p. 278, https://slac.stanford.edu/ pubs/slacreports/reports16/slac-r-839-b.pdf.

[3] M. Friedman, Autoacceleration of an Intense Relativistic Electron Beam, Phys. Rev. Lett. 31, 1107 (1973).

[4] E. A. Perevedentsev and A. N. Skrinsky, in Proceedings of 6th All-Union Conference Charged Particle Accelerators, Dubna (Institute of Nuclear Physics, Novosibirsk, USSR, 1978), Vol. 2, p. 272.

[5] A. M. Sessler, The free electron laser as a power source for a highgradient accelerating structure, AIP Conf. Proc. 91, 154 (1982).

[6] P. Chen, J. M. Dawson, R. W. Huff, and T. Katsouleas, Acceleration of Electrons by the Interaction of a Bunched Electron Beam with a Plasma, Phys. Rev. Lett. 54, 693 (1985).

[7] Y. Chin, The wakefield acceleration using a cavity of elliptical cross section, in Proceedings of Lin. Accef. Conference (LINAC '84), Seeheim, Federal Republic of Germany (1983), p. 159, https://accelconf.web.cern.ch/184/ papers/tup0026.pdf.

[8] W. Gai, P. Schoessow, B. Cole, R. Konecny, J. Norem, J. Rosenzweig, and J. Simpson, Experimental Demonstration of Wakefield Effects in Dielectric Structures, Phys. Rev. Lett. 61, 2756 (1988).

[9] K. L. Bane, P. Chen, and P. B. Wilson, On collinear wakefield acceleration, in Proceedings of the 11th Particle Accelerator Conference, PAC-1985: Accelerator Engineering and Technology (IEEE, New York, 1985), Vol. 32, p. 3524.

[10] S. S. Baturin and A. Zholents, Upper limit for the accelerating gradient in the collinear wakefield accelerator as a function of the transformer ratio, Phys. Rev. Accel. Beams 20, 061302 (2017).

[11] M. Cornacchia, S. Di Mitri, G. Penco, and A. A. Zholents, Formation of electron bunches for harmonic cascade X-ray free electron lasers, Phys. Rev. ST Accel. Beams 9, 120701 (2006).

[12] G. Penco, M. Danailov, A. Demidovich, E. Allaria, G. De Ninno, S. Di Mitri, W. M. Fawley, E. Ferrari, L. Giannessi, and M. TrovÃ, Experimental Demonstration of Electron Longitudinal-Phase-Space Linearization by Shaping the Photoinjector Laser Pulse, Phys. Rev. Lett. 112, 044801 (2014).

[13] F. Lemery and P. Piot, Tailored electron bunches with smooth current profiles for enhanced transformer ratios in beam-driven acceleration, Phys. Rev. ST Accel. Beams 18, 081301 (2015).

[14] G. Loisch, J. Good, M. Gross, H. Huck, I. Isaev, M. Krasilnikov, O. Lishilin, A. Oppelt, Y. Renier, F. Stephan, R. Brinkmann, F. Grüner, and I. Will, Photocathode laser based bunch shaping for high transformer ratio plasma wakefield acceleration, Nucl. Instrum. Methods Phys. Res., Sect. A 909, 107 (2018).

[15] I. Kuzmin, S. Mironov, E. Gacheva, V. Zelenogorsky, A. Potemkin, E. Khazanov, A. Kanareykin, S. Antipov, M.
Krasilnikov, G. Loisch, and F. Stephan, Shaping triangular picosecond laser pulses for electron photoinjectors, Laser Phys. Lett. 16, 015001 (2018).

[16] P. Piot, Y.-E. Sun, J. G. Power, and M. Rihaoui, Generation of relativistic electron bunches with arbitrary current distribution via transverse-to-longitudinal phase space exchange, Phys. Rev. ST Accel. Beams 14, 022801 (2011).

[17] B. Jiang, C. Jing, P. Schoessow, J. Power, and W. Gai, Formation of a novel shaped bunch to enhance transformer ratio in collinear wakefield accelerators, Phys. Rev. ST Accel. Beams 15, 011301 (2012).

[18] D. Y. Shchegolkov and E. I. Simakov, Design of an emittance exchanger for production of special shapes of the electron beam current, Phys. Rev. ST Accel. Beams 17, 041301 (2014).

[19] G. Ha, M. H. Cho, W. Namkung, J. G. Power, D. S. Doran, E. E. Wisniewski, M. Conde, W. Gai, W. Liu, C. Whiteford, Q. Gao, K.-J. Kim, A. Zholents, Y.-E. Sun, C. Jing, and P. Piot, Precision Control of the Electron Longitudinal Bunch Shape Using an Emittance-Exchange Beam Line, Phys. Rev. Lett. 118, 104801 (2017).

[20] Q. Gao, G. Ha, C. Jing, S. P. Antipov, J. G. Power, M. Conde, W. Gai, H. Chen, J. Shi, E. E. Wisniewski, D. S. Doran, W. Liu, C. E. Whiteford, A. Zholents, P. Piot, and S. S. Baturin, Observation of High Transformer Ratio of Shaped Bunch Generated by an Emittance-Exchange Beamline, Phys. Rev. Lett. 120, 114801 (2018).

[21] R. J. England, J. B. Rosenzweig, G. Andonian, P. Musumeci, G. Travish, and R. Yoder, Sextupole correction of the longitudinal transport of relativistic beams in dispersionless translating sections, Phys. Rev. ST Accel. Beams 8, 012801 (2005).

[22] P. Piot, C. Behrens, C. Gerth, M. Dohlus, F. Lemery, D. Mihalcea, P. Stoltz, and M. Vogt, Generation and Characterization of Electron Bunches with Ramped Current Profiles in a Dual-Frequency Superconducting Linear Accelerator, Phys. Rev. Lett. 108, 034801 (2012).

[23] T. K. Charles, D. M. Paganin, and R. T. Dowd, Causticbased approach to understanding bunching dynamics and current spike formation in particle bunches, Phys. Rev. Accel. Beams 19, 104402 (2016).

[24] J. Svensson, H. Ekerfelt, O. Lundh, E. Mansten, J. Andersson, M. Kotur, F. Lindau, S. Thorin, and T. Charles, Beamline design for plasma-wakefield acceleration experiments at MAX IV, in Proceedings of the 2018 IEEE Advanced Accelerator Concepts Workshop, ACC 2018 (Institute of Electrical and Electronics Engineers Inc., Breckenridge, Colorado, USA, 2019).

[25] G. Andonian, S. Barber, F. H. O'Shea, M. Fedurin, K. Kusche, C. Swinson, and J. B. Rosenzweig, Generation of Ramped Current Profiles in Relativistic Electron Beams Using Wakefields in Dielectric Structures, Phys. Rev. Lett. 118, 054802 (2017).

[26] Z. Dong, H. Chen, C. Song, Y. Tan, Q. Tian, H. Zhang, Z. Zhou, Z. Li, Y. Du, R. Li, L. Yan, W. Huang, and C. Tang, Longitudinal phase space manipulation with planar corrugated wakefield structures, Nucl. Instrum. Methods Phys. Res., Sect. A 987, 164819 (2021).

[27] P. Muggli, V. Yakimenko, M. Babzien, E. Kallos, and K. P. Kusche, Generation of Trains of Electron Microbunches 
with Adjustable Subpicosecond Spacing, Phys. Rev. Lett. 101, 054801 (2008).

[28] D. Shchegolkov, S. Antipov, M. Fedurin, and E. Simakov, Dielectric wakefield accelerator experiments at ATF, in Proceedings of 6th International Particle Accelerator Conference(IPAC'15), Richmond, VA, USA, May 3-8, 2015, International Particle Accelerator Conference No. 6 (JACoW, Geneva, Switzerland, 2015), pp. 2681-2684, https://doi.org/10.18429/JACoW-IPAC2015-WEPJE006.

[29] S. Schröder, K. Ludwig, A. Aschikhin, R. D’Arcy, M. Dinter, P. Gonzalez, S. Karstensen, A. Knetsch, V. Libov, C. A. Lindstrøm, F. Marutzky, P. Niknejadi, A. Rahali, L. Schaper, A. Schleiermacher, B. Schmidt, S. Thiele, A. de Zubiaurre Wagner, S. Wesch, and J. Osterhoff, Tunable and precise two-bunch generation at FLASHForward, J. Phys. Conf. Ser. 1596, 012002 (2020).

[30] G. Ha, J. G. Power, J. Shao, M. Conde, and C. Jing, Coherent synchrotron radiation free longitudinal bunch shaping using transverse deflecting cavities, Phys. Rev. Accel. Beams 23, 072803 (2020).

[31] W. K. H. Panofsky and M. Bander, Asymptotic theory of beam breakup in linear accelerators, Rev. Sci. Instrum. 39, 206 (1968).

[32] V. K. Neil, L. S. Hall, and R. K. Cooper, Further theoretical studies of the beam breakup instability, Part. Accel. 9, 213 (1979), http://cds.cern.ch/record/1107964.

[33] A. W. Chao, B. Richter, and C.-Y. Yao, Beam emittance growth caused by transverse deflecting fields in a linear accelerator, Nucl. Instrum. Methods 178, 1 (1980).

[34] V.E. Balakin, A. V. Novokhatsky, and V. P. Smirnov, VLEPP: Transverse beam dynamics, in Proceedings of the 12th International Conference on High-Energy Accelerators, HEACC 1983 (Fermilab, Batavia, 1983), Vol. C830811, p. 119.

[35] C. Li, W. Gai, C. Jing, J. G. Power, C. X. Tang, and A. Zholents, High gradient limits due to single bunch beam breakup in a collinear dielectric wakefield accelerator, Phys. Rev. ST Accel. Beams 17, 091302 (2014).

[36] D. Y. Shchegolkov, E. I. Simakov, and A. A. Zholents, Towards a practical multimeter long dielectric wakefield accelerator: Problems and solutions, IEEE Trans. Nucl. Sci. 63, 804 (2016).

[37] S. S. Baturin and A. Zholents, Stability condition for the drive bunch in a collinear wakefield accelerator, Phys. Rev. Accel. Beams 21, 031301 (2018).

[38] A. Zholents et al., A conceptual design of a compact wakefield accelerator for a high repetition rate multiuser $\mathrm{x}$ ray free-electron laser facility, in Proceedings of the 9th International Particle Accelerator Conference (IPAC'18), Vancouver, BC, Canada, 2018, International Particle Accelerator Conference No. 9 (JACoW Publishing, Geneva, Switzerland, 2018), pp. 1266-1268.

[39] G. J. Waldschmidt et al., Design and test plan for a prototype corrugated waveguide, in Proceedings of the 9th International Particle Accelerator Conference (IPAC'18), Vancouver, BC, Canada, 2018, International Particle Accelerator Conference (Ref. [38]) pp. 1550-1552.

[40] B. Zotter and S. Kheifets, Impedances and Wakes in High Energy Particle Accelerators (World Scientific Publishing Company, Singapore, 1998).
[41] A. Chao, Physics of Collective Beam Instabilities in High Energy Accelerators (Wiley and Sons, New York, 1993).

[42] A. Polyanin and A. Manzhirov, Handbook of Integral Equations (CRC Press, Boca Raton, 1998).

[43] A. E. Siy, G. J. Waldschmidt, and A. Zholents, Design of a compact wakefield accelerator based on a corrugated waveguide, in Proceedings of the 2019 North American Particle Accelerator Conference (NAPAC2019), Lansing, MI, 2019 (JACoW, Geneva, Switzerland, 2019), pp. 232-235.

[44] I. Zagorodnov, K. L. F. Bane, and G. Stupakov, Calculation of wakefields in 2D rectangular structures, Phys. Rev. ST Accel. Beams 18, 104401 (2015).

[45] J. Arthur et al., Linac Coherent Light Source (LCLS) conceptual design report, SLAC Report No. SLAC-R-593, Stanford Linear Accelerator Center, 2002.

[46] R. A. Bosch, K. J. Kleman, and J. Wu, Modeling two-stage bunch compression with wakefields: Macroscopic properties and microbunching instability, Phys. Rev. ST Accel. Beams 11, 090702 (2008).

[47] W. Tan, P. Piot, and A. Zholents, Longitudinal beamshaping simulation for enhanced transformer ratio in beamdriven accelerators, in 2018 IEEE Advanced Accelerator Concepts Workshop (AAC) (IEEE, Breckenridge, Colorado, USA, 2018), pp. 190-194, https://ieeexplore.ieee .org/xpl/conhome/8607198/proceeding.

[48] K. L. F. Bane and P. Emma, Litrack: A fast longitudinal phase space tracking code with graphical user interface, in Proceedings of the 21st Particle Accelerator Conference, Knoxville, TN, 2005 (IEEE, Piscataway, NJ, 2005), pp. 4266-4268.

[49] I. Zagorodnov, T. Weiland, and M. Dohlus, Wakefields generated by the LOLA-IV structure and the 3rd harmonic section in TTF-II, Technical Report No. TESLA Report 2004-01, DESY, Darmstadt, Germany, 2004.

[50] F.-A. Fortin, F.-M. De Rainville, M.-A. Gardner, M. Parizeau, and C. Gagné, DEAP: Evolutionary algorithms made easy, J. Machine Learning Res. 13, 2171 (2012), https://www.jmlr.org/papers/v13/fortin12a.html.

[51] T. Charles, M. Boland, R. Dowd, and D. Paganin, Beam by design: Current pulse shaping through longitudinal dispersion control, in Proceedings of the International Particle Accelerator Conference (IPAC'17), Copenhagen, Denmark, 2017, International Particle Accelerator Conference No. 8 (JACoW, Geneva, Switzerland, 2017), pp. 644-647.

[52] T. Xu, C. Jing, A. Kanareykin, P. Piot, and J. Power, Optimized electron bunch current distribution from a radio frequency photoemission source, in 2018 IEEE Advanced Accelerator Concepts Workshop (AAC) (IEEE, Breckenridge, Colorado, USA, 2018), pp. 199-203, https:// ieexplore.ieee.org/xpl/conhome/8607198/proceeding.

[53] K. Floettmann, ASTRA-A Space Charge Tracking Algorithm (Deutsches Elektronen-Synchrotron, Hamburg, Germany, 2017).

[54] J. Bisognano, R. Bosch, D. Eisert, M. Fisher, M. Green, K. Jacobs, K. Kleman, J. Kulpin, G. Rogers, J. Lawler, D. Yavuz, and R. Legg, Progress toward the Wisconsin free electron laser, Technical Report No. JLAB-ACC-11-1333, Jefferson Lab, 2011.

[55] J. Bisognano, M. Bissen, R. Bosch, M. Efremov, D. Eisert, M. Fisher, M. Green, K. Jacobs, R. Keil, K. Kleman, 
G. Rogers, M. Severson, D. D Yavuz, R. Legg, R. Bachimanchi, C. Hovater, T. Plawski, and T. Powers, Wisconsin SRF electron gun commissioning, in Proceedings of the 25th Particle Accelerator Conference, PAC2013, Pasadena, CA, 2013 (IEEE, New York, 2013), p. 622.

[56] R. Legg, W. Graves, T. Grimm, and P. Piot, Half wave injector design for WiFEL, in EPAC 2008-Contributions to the Proceedings (European Physical Society Accelerator Group, Genoa, Italy, 2008), pp. 469-471.

[57] W. H. Tan, P. Piot, and A. Zholents, Longitudinal-phasespace manipulation for efficient beam-driven structure wakefield acceleration, in Proceedings of the 10th International Particle Accelerator Conference (IPAC'19), Melbourne, Australia, 2019, International Particle Accelerator Conference (JACoW Publishing, Geneva, Switzerland, 2019), pp. 2296-2299.

[58] G. Nielsen, N. Hauge, E. Krauthammer, and A. Baurichter, Compact high-Tc $2 \mathrm{G}$ superconducting solenoid for superconducting rf electron gun, in Proceedings of the 4th International Particle Accelerator Conference (IPAC2013), Shanghai, China, 2013 (JACoW, Geneva, Switzerland, 2020), pp. 3514-3516.

[59] T. Xu, C.-J. Jing, A. Kanareykin, P. Piot, and J. Power, Spatiotemporal shaping of the photocathode laser pulse for low-emittance shaped electron bunches, in Proceedings of the 10th International Particle Accelerator Conference (IPAC'19), Melbourne, Australia, 2019, International Particle Accelerator Conference No. 10 (JACoW Publishing, Geneva, Switzerland, 2019), pp. 2163-2166.

[60] S. Gilevich, S. Alverson, S. Carbajo, S. Droste, S. Edstrom, A. Fry, M. Greenberg, R. Lemons, A. Miahnahri, W. Polzin, S. Vetter, and F. Zhou, The LCLS-II photoinjector drive laser system, in Proceedings of the Conference on Lasers and Electro-Optics (Optical Society of America, Washington DC, USA, 2020).

[61] G. Ferrini, P. Michelato, and F. Parmigiani, A Monte Carlo simulation of low energy photoelectron scattering in $\mathrm{Cs}_{2}$ Te, Solid State Commun. 106, 21 (1998).

[62] P. Piot, Y.-E. Sun, T. J. Maxwell, J. Ruan, E. Secchi, and J. C. T. Thangaraj, Formation and acceleration of uniformly filled ellipsoidal electron bunches obtained via spacecharge-driven expansion from a cesium-telluride photocathode, Phys. Rev. ST Accel. Beams 16, 010102 (2013).

[63] A. Aryshev, M. Shevelev, Y. Honda, N. Terunuma, and J. Urakawa, Femtosecond response time measurements of a Cs2Te photocathode, Appl. Phys. Lett. 111, 033508 (2017).

[64] V. Jain et al., $650 \mathrm{MHz}$ elliptical superconducting rf cavities for PIP-II project, in Proceedings, 2ndNorth American Particle Accelerator Conference (NAPAC2016) (JACoW, Chicago, Illinois, USA, 2017), https://doi.org/10.18429/JACoW-NAPAC2016WEB3CO03.

[65] F. Qi et al., Breaking 50 femtosecond Resolution Barrier in $\mathrm{MeV}$ Ultrafast Electron Diffraction with a Double Bend Achromat Compressor, Phys. Rev. Lett. 124, 134803 (2020).

[66] S.D. Mitri and M. Cornacchia, Transverse emittancepreserving arc compressor for high-brightness electron beam-based light sources and colliders, Europhys. Lett. 109, 62002 (2015).
[67] J. A. G. Akkermans, S. Di Mitri, D. Douglas, and I. D. Setija, Compact compressive arc and beam switchyard for energy recovery linac-driven ultraviolet free electron lasers, Phys. Rev. Accel. Beams 20, 080705 (2017).

[68] S. D. Mitri, Bunch length compressors, in Proceedings of the CAS-CERN Accelerator School on Free Electron Lasers and Energy Recovery Linacs (CERN, Geneva, Switzerland, 2018), Vol. 1, p. 363.

[69] A. Chao, K. H. Mess, M. Tigner, and F. Zimmermann, Handbook of Accelerator Physics and Engineering, 2nd ed. (World Scientific, Singapore, 2013).

[70] D. Robin, E. Forest, C. Pellegrini, and A. Amiry, Quasiisochronous storage rings, Phys. Rev. E 48, 2149 (1993).

[71] P. H. Williams, G. Pérez-Segurana, I. R. Bailey, S. Thorin, B. Kyle, and J. B. Svensson, Arclike variable bunch compressors, Phys. Rev. Accel. Beams 23, 100701 (2020).

[72] N. Sudar, Y. Nosochkov, K. Bane, Z. Zhang, and Y. Ding, Octupole based current horn suppression in multistage bunch compression with emittance growth correction, Phys. Rev. Accel. Beams 23, 112802 (2020).

[73] P. Emma and R. Brinkmann, Emittance dilution through coherent energy spread generation in bending systems, in Proceedings of the Particle Accelerator Conference, Vancouver, BC, Canada, 1997 (IEEE, New York, 1997), Vol. 2, pp. 1679-1681, Cat. No. 97CH36167.

[74] E. Saldin, E. Schneidmiller, and M. Yurkov, Longitudinal space charge-driven microbunching instability in the TESLA Test Facility linac, Nucl. Instrum. Methods Phys. Res., Sect. A 528, 355 (2004).

[75] Z. Huang, M. Borland, P. Emma, J. Wu, C. Limborg, G. Stupakov, and J. Welch, Suppression of microbunching instability in the linac coherent light source, Phys. Rev. ST Accel. Beams 7, 074401 (2004).

[76] Y.S. Derbenev, J. Rossbach, E. L. Saldin, and V. D. Shiltsev, Microbunch radiative tail-head interaction, Technical Report No. TESLA-FEL 1995-05, DESY, 1995.

[77] G. Huang et al., High precision rf control for the LCLS-II, in Proceedings of the 3rd North American Particle Accelerator Conference (NAPAC'16), Chicago, IL, 2016, North American Particle Accelerator Conference No. 3 (JACoW, Geneva, Switzerland, 2017), pp. 1292-1296.

[78] T. A. Mohayai, P. Snopok, D. Neuffer, and C. Rogers, Novel application of density estimation techniques in muon ionization cooling experiment, in Proceedings, Meeting of the APS Division of Particles and Fields (DPF 2017) (APS, Fermilab, Batavia, Illinois, USA, 2017), http://lss.fnal.gov/archive/2017/conf/fermilabconf-17-476-apc.pdf.

[79] J. Qiang, R. D. Ryne, M. Venturini, A. A. Zholents, and I. V. Pogorelov, High resolution simulation of beam dynamics in electron linacs for x-ray free electron lasers, Phys. Rev. ST Accel. Beams 12, 100702 (2009).

[80] E. L. Saldin, E. A. Schneidmiller, and M. V. Yurkov, On the coherent radiation of an electron bunch moving in an arc of a circle, Nucl. Instrum. Methods Phys. Res., Sect. A 398, 373 (1997).

[81] C. E. Mitchell, J. Qiang, and R. D. Ryne, A fast method for computing 1-D wakefields due to coherent synchrotron radiation, Nucl. Instrum. Methods Phys. Res., Sect. A 715, 119 (2013). 